\title{
Helicon waves in a flaring magnetic field
}

\author{
Donald Arnush $\dagger$ and Arthur Peskoff $\ddagger$ \\ $\dagger$ Electrical Engineering Department, UCLA School of Engineering and Applied Science, Los \\ Angeles, CA 90095-1594, USA \\ $\ddagger$ Departments of Biomathematics and Physiology, UCLA School of Medicine, Los Angeles, \\ CA 90095-1766, USA
}

Received 19 April 1995, in final form 26 September 1995

\begin{abstract}
Helicon wave (whistler waves bounded transversely by a magnetic field surface) propagation is investigated for a cylindrically symmetric curved (flaring) boundary using a finite-element method where the propagation region is divided into a sequence of truncated cones. In each conical segment, a local spherical coordinate system is used with the origin at the apex of the cone. A vector wave equation for the fields is formulated for a cold plasma and reduced, in spherical coordinates, to a pair of coupled partial differential equations (PDEs) for two scalar functions. The PDEs are separable for the azimuthal eigenvalue $m=0$. The $\boldsymbol{\theta}$-dependence is a Legendre function, with non-integer eigenvalues determined by the cone angle. The dependence on the radial coordinate, $x$, satisfies a fourth-order ordinary differential equation (ODE). A straightforward numerical integration of the equation from $x_{1}$ to $x_{2}$ fails for large values of $x_{2}-x_{1}$, because of the existence of an exponentially growing solution to the equation. Consequently, a different approach is needed. Four independent solutions, valid for $0 \leqslant x<\infty$, in the form of power series in $x$ (PS) are obtained which are each asymptotically proportional to $\mathrm{e}^{x}$ as $x \rightarrow \infty$. For $x$ large, four asymptotic expansions (AE) are obtained in the form of series in $1 / x$ times $\mathrm{e}^{ \pm \mathrm{i} x}$ or $\mathrm{e}^{ \pm x}$. The problem then is to find four linear combinations of the four PSs, each of which approaches one of the AEs in the limit $x \rightarrow \infty$ by first deriving a double-integral representation of each PS, valid for all $x$, and then using its $x \rightarrow \infty$ limit to match each AE to a linear combination of the PSs. For special values of $\ell(\ell=4 N-1$ or $4 N, N$ an integer) closed-form solutions (polynomials times exponentials) result that are exact representations of the solution for all $x$. Solutions are computed and illustrated for an outgoing wave using the closed-form solution for special values of $\ell$, and as a function of $\ell$ and $x$ using the PS and AE, which have a large range of overlap. The propagating solutions of the ODE are also obtained using a WKB method and are used to calculate the propagation in a slowly diverging parabola of revolution.
\end{abstract}

\section{Introduction}

Low-frequency electromagnetic whistler waves are well known in ionospheric and laboratory research. When transversely confined, they are often called 'helicon' waves. They develop electrostatic components and their propagation and polarization characteristics are modified. An extensive review of the experimental and theoretical research on the properties of helicon waves confined to a cylinder was performed by Chen (1994). He discussed the unusually high plasma production efficiency, uniformity, and quiescence of helicon plasma sources, and their consequent suitability for semiconductor processing and other industrial applications.

In this paper we find exact solutions for helicon waves in conical regions, which can be used in a finite-element method for analysing the propagation of helicon waves in a 
flaring static guide field. To be able to calculate propagation in an arbitrary flaring field, exact solutions valid in each element are obtained. A simplified WKB approximation is also obtained and compared to the exact solution to provide one criterion for its range of validity. As an illustration, the method is used to calculate propagation in a guide field which flares slowly enough to use the WKB method.

In both ionospheric and industrial cases, helicon waves are usually confined to regions bounded by curved static magnetic field $\left(\boldsymbol{B}_{0}\right)$ line surfaces. Although theoretical and experimental treatments of cylindrically confined helicon waves are a vigorous ongoing research subject, there is very little experimental or theoretical published research on helicon waves confined in a non-uniform field geometry. In section 2 of this paper we consider that case by first formulating a helicon vector wave equation for an arbitrary $\boldsymbol{B}_{0}$. We anticipate calculating the propagation in an axisymmetric guide field by employing a method (see, e.g., Sporleder and Unger 1979 or Solymar 1959) commonly used for calculating microwave propagation in horns and couplers. For guided waves in vacuum, authors usually segment the enclosed region into either a sequence of cylinders, or a sequence of lenticular regions with coaxial conical sides and spherical surfaces perpendicular to the axis. In this paper, where a plasma-filled guide is considered, we use the latter approach. In each lenticular region we employ spherical coordinates with the origin at the apex of the tangent cone. The magnetic field lines then coincide with radius vectors on the conical surfaces and the axis, and are approximated by them in between.

The helicon vector wave equation is reduced to a pair of coupled equations for two scalar functions in the case that the elements of the dielectric tensor are functions only of the spherical radius in each lenticular region (i.e. there is no transverse dependence). Assuming, along with Chen (1991), that the normal component of the wave current vanishes at the conical surfaces, we find solutions of the coupled equations that are separable in spherical coordinates for azimuthally symmetric waves $(m=0)$. The $\vartheta$-dependence is described in terms of Legendre functions of non-integer eigenvalue $\ell$. The two coupled radial equations can be reduced to a single fourth-order ordinary differential equation (ODE, see equation (17)) for the radial functions, $H_{\ell}$, with complicated coefficients. The separation can also be made for the small cone angle limit, and arbitrary $m$. The ODE is then considered for the frequently encountered intermediate frequency case

$$
\omega_{\mathrm{ci}} \ll \omega \ll \omega_{\mathrm{ce}} \ll \omega_{\mathrm{pe}}
$$

where $\omega, \omega_{\mathrm{c}}$ and $\omega_{\mathrm{p}}$ are the wave, cyclotron, and plasma frequencies, respectively, and $\mathrm{i}$ and e refer to ions and electrons.

In this approximation displacement current is negligible, as is the electric wave field along the guide field (due to high conductivity), and the wave current is primarily Hall current. To be specific, we consider a configuration which occurs in many experiments and processing tools in which a steady-state plasma is produced in a high magnetic field region where it has very high mobility along the field lines and low mobility transversely. The plasma is assumed to expand freely along a flaring magnetic field into a large volume. Since the conserved particle flux is directed along the field lines, its divergence, as well as that of $\boldsymbol{B}_{0}$, vanishes. Assuming a constant flow velocity, $n_{0} / B_{0}$ is a constant $\left(n_{0}\right.$ is the electron density). As the plasma expands, $B_{0}$, and hence $\omega_{\mathrm{ce}}$, decreases until $\omega_{\mathrm{ce}}$ approaches $\omega$, the electrons approach cyclotron resonance and the second inequality of equation (1) is no longer valid. In most cases of practical interest ionic effects are unimportant and the first inequality of equation (1) is valid. Of course, since the coordinate system is fitted to the boundary field lines and the magnetic field is divergence free, the lenticular regions need never include the origin. Although the fourth-order ODE is more generally 
applicable, we restrict our considerations in the remainder of the paper to regions where inequalities (1) obtain, and we scale the radius, $r$, in each region to the whistler wavenumber in an unbounded medium $q=\left(\omega_{\mathrm{p}} / c\right)\left(\omega / \omega_{\mathrm{c}}\right)^{1 / 2}$, i.e. $x=q r$ is the non-dimensional radial variable.

In section 3 we address the general case where it is necessary to follow the solution over the entire range of the non-dimensional radial coordinate $x$ to calculate it accurately for all cases of practical interest. The direct numerical approach to solving an initialvalue problem by integrating the equation numerically, for example, using the RüngeKutta method (Dahlquist and Björck 1974) does not work in the present case because there is a solution to the equation that grows exponentially with $x$. Consequently, any error in the initial value, or in the numerical computation, will eventually be amplified exponentially as $x$ increases and will overshadow the desired solution. Our attempts to solve the equation numerically on a PC in double precision (16 digits) failed for radial distances $\left(x_{2}-x_{1}\right) \gtrsim 20$, and so a different approach must be taken.

Instead of direct numerical integration, we find analytic functions which represent the solutions to the equation. The usual special functions that arise in mathematical physics (e.g. Bessel, Legendre and hypergeometric functions) are solutions to second-order differential equations. For the present fourth-order equation, no such previously obtained solutions are available.

In section 3.1, we find four power series solutions to the ODE, equation (17), which converge for all $x$ using the method of Frobenius (Morse and Feshbach 1953). Each of these four series is asymptotically proportional to $\mathrm{e}^{x}$ for large $x$. However, for large $x$, equation (17) has four independent solutions that approach $\mathrm{e}^{ \pm \mathrm{ix}}$ and $\mathrm{e}^{ \pm x}$. For physical problems of interest we want to obtain outgoing and incoming wave solutions $\left(\mathrm{e}^{ \pm \mathrm{i} x}\right)$ as $x \rightarrow \infty$ which are valid for all $x$. Consequently, we must remove the exponentially growing components from the Frobenius solutions. This is done by finding four linear combinations of the four series solutions which approach $\mathrm{e}^{ \pm \mathrm{i} x}$ and $\mathrm{e}^{ \pm x}$, respectively, as $x \rightarrow \infty$. In section 3.2 , we obtain four asymptotic expansions for the solutions to equation (17), in the form of series in inverse powers of the normalized radial distance $x$ times $\mathrm{e}^{ \pm \mathrm{i} x}$ or $\mathrm{e}^{ \pm x}$. For particular integer values of the eigenvalue $\ell(\ell=4 N, 4 N-1 ; N=1,2, \ldots)$, the series terminates after $\ell-2$ terms and the asymptotic result becomes exact for all $x$. In section 3.3, doubleintegral representations of the four series solution are obtained. In section 3.4, by asymptotic evaluation of the integral representations in the limit of large $|x|$, the connection between the convergent series expansions and the large- $x$ asymptotic expansions is made. It yields four linear combinations of the four series solutions that approach $\mathrm{e}^{ \pm \mathrm{ix}}$ and $\mathrm{e}^{ \pm x}$, respectively. The general solution can be computed using the appropriate linear combination of the series solutions for small $x$ and the asymptotic expansions for large $x$. Results of the computation are shown for outgoing waves.

In section 5 a WKB approximation to equation (17) is obtained and compared to the exact solution. A sufficient condition for the validity of the WKB approximation is $k^{\prime} / k \ll k$, where $k$ is the WKB wavenumber and $k^{\prime}$ its derivative with respect to $r$. The condition that the rate of flare of a guide field be sufficiently gentle to render reflections negligible has the same form, except that the derivative is with respect to the axial distance, $z$. As an illustrative example, we calculate the helicon wave field emanating from a source in a finite solenoid with end windings arranged such that it produces a cylinder-like guide field followed by a flaring field which can be approximated near the solenoid by a parabola of revolution. We follow the waves for as long as both the WKB and the conditions of equation (1) are met. 


\section{Formulation}

For concreteness, consider figure 1, where we index each truncated conical element by the $z$-coordinate of its left-hand boundary circle. Consider a wave incident at $z=0$ from a cylindrical guide on the left. Conical waves propagate from element to element, with all components of their vector fields continuous at the spherical interfaces. At the spherical surface separating regions $n$ and $n+1$, to ensure that the fields be continuous as a function of $\vartheta$, the field solution of the $n$ region can be expanded in spherical harmonics of the $n+1$ region. Thus, a spectrum of $\ell$-modes is generated. For each $\ell$-mode, to ensure field continuity in the radial direction, $H_{\ell}, H_{\ell}^{\prime}, H_{\ell}^{\prime \prime}$ and $H_{\ell}^{\prime \prime \prime}$ must be continuous and, in the general case, all four solutions of the ODE have to be employed to satisfy the four conditions. On physical grounds we expect that for guide fields approximated by cones with slowly changing angles the forward propagating solution dominates. In the more general case the backward propagating reflected wave and the two exponential solutions provide corrections necessary to account for the curvature of the transverse boundary.

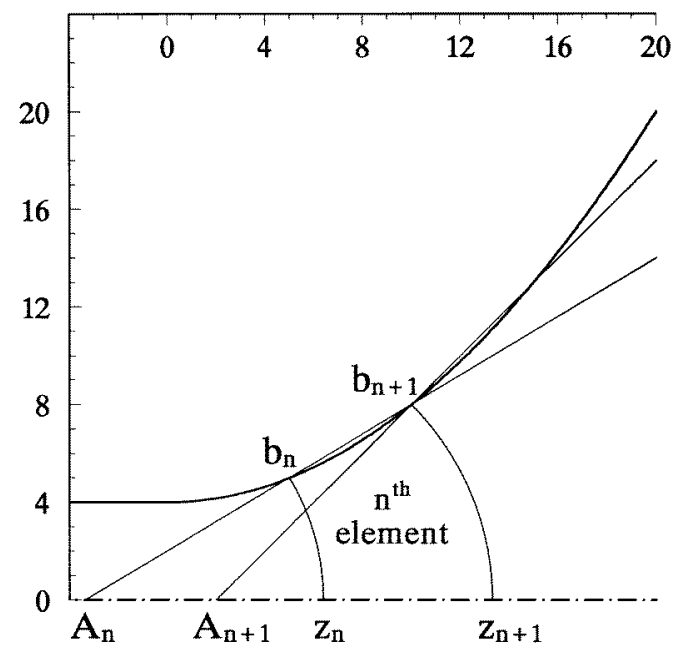

Figure 1. Construction of a finite element (conical segmentation) in flaring fields. In a plane which includes the axis of symmetry, $z$, boundary points $b_{n}$ are selected at uniform $z$-coordinate intervals (for example). $A_{n}$ is the intersection of the $z$-axis and the line which includes $b_{n}$ and $b_{n+1}$. The $n$th conical segment is bounded by the cone with its apex at $A_{n}$, and the spherical surfaces with centres at $A_{n}$, and radii given by the distances $\left(A_{n}, b_{n}\right)$ and $\left(A_{n}, A_{n+1}\right)+\left(A_{n}, b_{n+1}\right)$.

For $z \leqslant 0$ (in the cylinder) we have an incident and a reflected wave, and a solution which decreases exponentially to the left. In the interior elements, we have all four solutions. If there are $N$ interfacial surfaces in element $N+1$, which includes the point at infinity, we have an outgoing wave and a wave that decreases exponentially to the right. For a unit incident wave, there are $4 N$ functions and $4 N$ equations corresponding to the conditions at the surfaces. If we terminate the angular eigenfunction series at $L$, it is necessary to solve $4 L N$ simultaneous equations in $4 L N$ unknowns.

\subsection{Vector wave equation}

We form an orthogonal curvilinear coordinate system $\xi_{1}, \xi_{2}, \xi_{3}$, where $\hat{\boldsymbol{\xi}}_{3}$ is the unit vector along $\xi_{3}$. Assuming $\exp (-\mathrm{i} \omega t)$ time dependence, most of the dominant physical properties of helicon waves can be obtained using the dielectric tensor for a cold collisional plasma (see e.g. Stix 1992),

$$
\varepsilon=\left|\begin{array}{ccc}
S & -\mathrm{i} D & O \\
\mathrm{i} D & S & 0 \\
0 & 0 & P
\end{array}\right|
$$


where the three tensor directions at any point coincide with the three orthogonal curvilinear coordinates, and

$$
\begin{aligned}
S & =\frac{1}{2}(R+L)=1-\sum_{j} \frac{\omega_{\mathrm{p} j}^{2}\left(\omega+\mathrm{i} v_{j}\right)}{\omega\left[\left(\omega+\mathrm{i} v_{j}\right)^{2}-\omega_{\mathrm{c} j}^{2}\right]} \\
D & =\frac{1}{2}(R-L)=\sum_{j} \frac{\varepsilon_{j} \omega_{c j} \omega_{\mathrm{p} j}^{2}}{\omega\left(\left(\omega+\mathrm{i} v_{j}\right)^{2}-\omega_{\mathrm{c} j}^{2}\right)} \\
P & =1-\sum_{j} \frac{\omega_{\mathrm{p} j}^{2}}{\omega\left(\omega+\mathrm{i} v_{j}\right)}
\end{aligned}
$$

where $j$ is the species, $\varepsilon_{j}$ the sign of its charge and $v_{j}$ is the collision frequency. Using equations (2), (3), and Maxwell's equations, we readily obtain

$$
\nabla \times \boldsymbol{B} / \mu_{0}=\boldsymbol{J}-\mathrm{i} \omega \varepsilon_{0} \boldsymbol{E}=\boldsymbol{J}_{T}=-\mathrm{i} \omega \varepsilon_{0}\left[S \boldsymbol{E}_{\perp}+P E_{3} \hat{\boldsymbol{\xi}}_{3}+\mathrm{i} D \hat{\boldsymbol{\xi}}_{3} \times \boldsymbol{E}\right]
$$

where $\boldsymbol{E}_{\perp}=\boldsymbol{E}-E_{3} \hat{\boldsymbol{\xi}}_{3}$. Equation (4) can be inverted to solve for $\boldsymbol{E}$ in terms of $\nabla \times \boldsymbol{B}$. Using i $\omega \boldsymbol{B}=\nabla \times \boldsymbol{E}$, we obtain

$$
\begin{aligned}
& \boldsymbol{E}=\frac{\mathrm{i}}{\omega \varepsilon_{0}}\left[\mathrm{i} \beta_{1} \hat{\boldsymbol{\xi}}_{3} \times \boldsymbol{J}_{T}+\left(\beta_{3}-\beta_{2}\right) \boldsymbol{J}_{T}+\beta_{3} \hat{\boldsymbol{\xi}}_{3} \times\left(\hat{\boldsymbol{\xi}}_{3} \times \boldsymbol{J}_{T}\right)\right] \\
& k_{0}^{2} \boldsymbol{B}=\nabla \times\left[\mathrm{i} \beta_{1} \hat{\boldsymbol{\xi}}_{3} \times(\nabla \times \boldsymbol{B})-\beta_{2}(\nabla \times \boldsymbol{B})+\beta_{3}(\nabla \times \boldsymbol{B})_{3} \hat{\boldsymbol{\xi}}_{3}\right]
\end{aligned}
$$

where

$$
\begin{aligned}
& k_{0}=\frac{\omega}{c} \quad \beta_{1}=\frac{L-R}{2 R L}=\frac{D}{D^{2}-S^{2}} \\
& \beta_{2}=-\frac{R+L}{2 R L}=\frac{S}{D^{2}-S^{2}} \quad \text { and } \quad \beta_{3}=\frac{1}{P}+\beta_{2} .
\end{aligned}
$$

The coefficients $\beta_{1}$ and $\beta_{3}-\beta_{2}$ are dimensionless Hall and conduction impedances, respectively. The third coefficient, $\beta_{3}$, vanishes in the absence of displacement current. In vacuum, $\beta_{1}=\beta_{3}=0, \beta_{2}=-1$, and equation (5) reduces to the vector Helmholtz equation $\left(\nabla^{2} \boldsymbol{B}+k_{0}^{2} \boldsymbol{B}=0\right)$. For intermediate frequencies typical of laboratory values $\left(n_{0}=10^{13} \mathrm{~cm}^{-3}, B_{0}=1 \mathrm{kG}\right.$ and $\omega / 2 \pi=27 \mathrm{MHz}$ and hence $\omega=1.7 \times 10^{8}, \omega_{\mathrm{ce}}=$ $1.8 \times 10^{10}$ and $\omega_{\mathrm{pe}}=1.8 \times 10^{11} \mathrm{rad} \mathrm{s}^{-1}$ ), the inequalities in equation (1) obtain, and

$$
\beta_{1} \cong \frac{\omega \omega_{\mathrm{ce}}}{\omega_{\mathrm{pe}}^{2}} \cong 10^{-4} \quad \beta_{2} \cong \frac{\omega}{\omega_{\mathrm{ce}}} \beta_{1} \quad \text { and } \quad \beta_{3} \cong \beta_{2}^{2} .
$$

Hence, $\beta_{1} \gg \beta_{2} \gg \beta_{3}$. Note that in this limit $\beta_{1}$ is independent of collisions. At lower magnetic fields, in the $\omega_{\mathrm{ce}} \rightarrow \omega$ limit, $\beta_{2}$ approaches $\beta_{1}$ but $\beta_{3}$ remains negligible.

\subsection{Vector equation separation in spherical coordinates}

We employ the multipolar potential method, used by Morse and Feshback (1953) to separate the vector Helmholtz equation in five confocal quadratic coordinate systems, by applying it to equation (5) in spherical coordinates and assuming that

$$
\boldsymbol{B}=\boldsymbol{M}+\boldsymbol{N} \quad \boldsymbol{M}=\nabla \times(\hat{\boldsymbol{r}} r \psi) \quad \text { and } \quad \boldsymbol{N}=\nabla \times \nabla \times(\hat{\boldsymbol{r}} r \chi) .
$$

Since $\hat{\boldsymbol{r}} \cdot \boldsymbol{M}=0$ and $\boldsymbol{r} \cdot \nabla \times \boldsymbol{N}=0, \boldsymbol{M}$ and $\boldsymbol{N}$ are interpreted as transverse magnetic and transverse electric wave fields, respectively. For the vector Helmholtz equation, $M$ and $N$ and hence $\psi$ and $\chi$ are decoupled, and each scalar function satisfies the scalar Helmholtz equation, e.g. $\nabla^{2} \psi+k_{0}^{2} \psi=0$. For the helicon wave equation, we insert equation (7) into 
equation (5), and find that $M$ and $N$ are coupled. The insertion yields an equation of the form

$\nabla \times \boldsymbol{P}(\psi, \chi, \hat{\boldsymbol{r}})=0$

$\boldsymbol{P}=\left[\mathrm{i} \beta_{1} \hat{\boldsymbol{r}} \times(\nabla \times \boldsymbol{B})-\beta_{2}(\nabla \times \boldsymbol{B})+\beta_{3} \hat{\boldsymbol{r}} \cdot(\nabla \times \boldsymbol{B}) \hat{\boldsymbol{r}}\right]-k_{0}^{2}[\hat{\boldsymbol{r}} r \psi+\nabla \times(\hat{\boldsymbol{r}} r \chi)]$.

Equation (8) is solved if we can demonstrate that there exists a scalar function, $f$, such that

$$
\boldsymbol{P}(\psi, \chi, \hat{\boldsymbol{r}})=-\nabla f \text {. }
$$

Equating the vector components of equation (9), we find that it is satisfied for

$$
f=\mathrm{i} \beta_{1} r \nabla^{2} \chi+\beta_{2} \frac{\partial}{\partial r}(r \psi) .
$$

Using this relation to eliminate $f$ from the component equations, we obtain

$k_{0}^{2} r \psi=\frac{\partial}{\partial r}\left(\mathrm{i} \beta_{1} r \nabla^{2} \chi\right)+\beta_{2} r \nabla^{2} \psi+\frac{\mathrm{d} \beta_{2}}{\mathrm{~d} r} \frac{\partial}{\partial r}(r \psi)-\beta_{3}\left[r \nabla^{2} \psi-\frac{\partial^{2}}{\partial r^{2}}(r \psi)\right]$

$k_{0}^{2} r \chi=\mathrm{i} \beta_{1} \frac{\partial}{\partial r}(r \psi)+\beta_{2} r \nabla^{2} \chi$.

The $\beta_{1}, \beta_{2}$, and $\beta_{3}$, and hence the plasma parameters, may be functions of $r$, but not of $\vartheta$ or $\varphi$. In the intermediate frequency approximation of equation (1) this condition requires that $n_{0} / B_{0}$ be a function of $r$ only. Thus, in contrast to the usual treatments of cylindrically confined waves in which exponential $z$ dependence of the fields is assumed from the start, the equation is separable for plasma parameter variation along, but not transverse to, the direction of wave propagation. Since $\vartheta$ and $\varphi$ derivatives in equations $(10 a)$ and $(10 b)$ appear only as $\nabla^{2}$, we expand $\psi$ and $\chi$ in the angular eigenfunctions of $\nabla^{2}$, the spherical harmonics $Y_{\ell, m}(\vartheta, \varphi)=P_{\ell}^{m}(\cos \vartheta) \exp (\operatorname{im} \varphi)$, where $P_{\ell}^{m}(\cos \vartheta)$ is the Legendre function:

$r \psi=\sum_{\ell, m} F_{\ell, m}(r) Y_{\ell, m}(\vartheta, \varphi) \quad$ and $\quad r \chi=\sum_{\ell, m} G_{\ell, m}(r) Y_{\ell, m}(\vartheta, \varphi)$.

The separation constants $\ell$ and $m$ are determined by the boundary conditions. Since $0 \leqslant \varphi<2 \pi$, and the fields are continuous, $m$ is an integer and the $m$-modes are independent. Defining the operator $D_{\ell}^{2}$

$$
D_{\ell}^{2}=\frac{\mathrm{d}^{2}}{\mathrm{~d} r^{2}}-\frac{\ell(\ell+1)}{r^{2}}
$$

inserting equation (11) in equations (10), using the second-order differential equations that $\exp (\mathrm{i} m \varphi)$ and $P_{\ell}^{m}(\cos \vartheta)$ satisfy, and the orthogonality of the $\exp (\operatorname{im} \varphi)$ to eliminate the sum over $m$, we obtain

$$
\begin{aligned}
& \sum_{\ell}\left\{k_{0}^{2} F_{\ell, m}-\beta_{2} D_{\ell}^{2} F_{\ell, m}-\beta_{2}^{\prime} F_{\ell, m}^{\prime}-\beta_{3} \frac{\ell(\ell+1)}{r^{2}} F_{\ell, m}-\left(\mathrm{i} \beta_{1} D_{\ell}^{2} G_{\ell, m}\right)^{\prime}\right\} P_{\ell}^{m}=0 \\
& \sum_{\ell}\left\{k_{0}^{2} G_{\ell, m}-\beta_{2} D_{\ell}^{2} G_{\ell, m}+\mathrm{i} \beta_{1} F_{\ell, m}^{\prime}\right\} P_{\ell}^{m}=0
\end{aligned}
$$

where the primes denote differentiation with respect to $r$. We assume that for each $m$, the component of the current perpendicular to the boundary, $J_{\vartheta}$, vanishes. Using equations (4), (5), (7), and (11), we obtain at the conical boundary, $\vartheta=\vartheta_{0}$,

$$
\begin{aligned}
& \mu_{0} r J_{\vartheta}=\sum_{\ell}\{ {\left[\left(1+\beta_{2}\right) \frac{\mathrm{d}}{\mathrm{d} \vartheta} P_{\ell}^{m}-\frac{m}{\sin \vartheta} \beta_{1} P_{\ell}^{m}\right] F_{\ell, m}^{\prime} } \\
&\left.+\mathrm{i}\left[\beta_{1} \frac{\mathrm{d}}{\mathrm{d} \vartheta} P_{\ell}^{m}-\left(1+\beta_{2}\right) \frac{m}{\sin \vartheta} P_{\ell}^{m}\right] D_{\ell}^{2} G_{\ell, m}\right\}\left.\right|_{\vartheta=\vartheta_{0}}=0 .
\end{aligned}
$$


For separability, it is required that there exists a set of eigenvalues of $\ell$, such that for each eigenvalue, the curly-bracketed expression in the boundary condition (14) vanishes for all $r$. For these eigenvalues of $\ell$, the $P_{\ell}^{m}(\cos \vartheta)$ would then be an orthogonal set of eigenfunctions. As a consequence of the orthogonality, the expressions in curly brackets in equations (13a) and (13b), for each $\ell$, must vanish, leading to a pair of coupled differential equations for $F_{\ell, m}$ and $G_{\ell, m}$. The expression in curly brackets in equation (14) does not separate into a function of $r$ times a function of $\vartheta$, unless $F_{\ell, m} / D_{\ell}^{2} G_{\ell, m}$ is independent of $r$. But this can be seen to be impossible if $F_{\ell, m}$ and $G_{\ell, m}$ are solutions of the coupled differential equations arising from equations (13). (The functions $F$ and $G$ would be overprescribed: three non-redundant equations for two unknown functions.) Thus, separability can occur only if both expressions in square brackets in equation (14) vanish simultaneously for all $r$. This is in turn possible if either

$\beta_{1}=1+\beta_{2} \quad$ and $\left.\quad\left[\frac{\mathrm{d}}{\mathrm{d} \vartheta} P_{\ell}^{m}(\cos \vartheta)-\frac{m}{\sin \vartheta} P_{\ell}^{m}(\cos \vartheta)\right]\right|_{\vartheta=\vartheta_{0}}=0$

or

$m=0 \quad$ and $\left.\quad \frac{\mathrm{d}}{\mathrm{d} \vartheta} P_{\ell}^{m}(\cos \vartheta)\right|_{\vartheta=\vartheta_{0}}=0$

are satisfied. The condition $\beta_{1}=1+\beta_{2}$ in equation $(15 a)$ is not physically realizable for the intermediate frequency case equation (1). Consequently, separability is only achievable for the azimuthally symmetric case $m=0$. In effect, the transverse electric and magnetic modes satisfy functionally different conditions at the boundary, and their coupling by the helicon interaction prevents the general simultaneous satisfaction of both. For fixed $\ell$, in the large- $r$ limit, $G_{\ell} / r^{2}$ can be neglected, $F_{\ell}^{\prime}$ and $G_{\ell}^{\prime \prime}$ approach proportionality and hence the equation can be satisfied approximately for each $\ell$. The result might be anticipated from the fact that for large $r$ and fixed $r \vartheta_{0}$, a conical segment approaches a segment of a cylinder in which higher order modes are separable. For further insights from comparison with the cylindrical case, including a generalization of the familiar helicon dispersion relation of Chen (1991), see the appendix. Unlike the case where $P_{\ell}^{m}(\cos \vartheta)$ covers the domain of $0 \leqslant \vartheta \leqslant \pi$, the eigenvalues, $\ell$, are not integers. The goal of the remainder of this effort is to analyse the axisymmetric case $m=0$.

Setting $m=0$, and using the orthogonality of the $P_{\ell}$ in equations (13a) and (13b), we obtain

$$
\begin{aligned}
& k_{0}^{2} F_{\ell}-\beta_{2} D_{\ell}^{2} F_{\ell}-\beta_{2}^{\prime} F_{\ell}^{\prime}-\beta_{3} \frac{\ell(\ell+1)}{r^{2}} F_{\ell}-\left(\mathrm{i} \beta_{1} D_{\ell}^{2} G_{\ell}\right)^{\prime}=0 \\
& k_{0}^{2} G_{\ell}-\beta_{2} D_{\ell}^{2} G_{\ell}+\mathrm{i} \beta_{1} F_{\ell}^{\prime}=0 .
\end{aligned}
$$

$F_{\ell}$ can be eliminated from equations $(13 c)$ and $(13 d)$ to obtain a single fourth-order ODE which is valid for any $\beta_{1}, \beta_{2}$ and $\beta_{3}$ which are functions of $r$ only.

\subsection{Intermediate frequency $m=0$ waves}

We now proceed to investigate the solutions to equations $(13 c)$ and $(13 d)$ in the intermediate frequency regime of equation (1) for $m=0$. Neglecting $\beta_{1}$ and $\beta_{2}$, defining the function $H_{\ell}=D_{\ell}^{2} G_{\ell}$, and eliminating $F_{\ell}=\mathrm{i}\left(\beta_{1} H_{\ell}\right)^{\prime} / k_{0}^{2}$, equations $(13 c)$ and $(13 d)$ can be reduced to the single equation

$$
\frac{\mathrm{d}^{2}}{\mathrm{~d} r^{2}}\left[\beta_{1} \frac{\mathrm{d}^{2}}{\mathrm{~d} r^{2}}\left(\beta_{1} H_{\ell}\right)\right]-\frac{\ell(\ell+1)}{r^{2}} \beta_{1} \frac{\mathrm{d}^{2}}{\mathrm{~d} r^{2}}\left(\beta_{1} H_{\ell}\right)-k_{0}^{4} H_{\ell}=0 .
$$


To explore the solutions to equation (16), we assume, as explained in the introduction, that there is no plasma production and negligible transverse plasma transport in the flaring field. In that case, we expect that $\beta_{1} \propto B_{0} / n_{0}$ will be constant. Equation (16) then takes the deceptively simple form

$$
\frac{\mathrm{d}^{4}}{\mathrm{~d} x^{4}} H_{\ell}-\frac{\ell(\ell+1)}{x^{2}} \frac{\mathrm{d}^{2}}{\mathrm{~d} x^{2}} H_{\ell}-H_{\ell}=0
$$

where we have used the general definition of $q=k_{0} / \beta_{1}^{1 / 2}$. The fields take on a simple form in terms of $H$ :

$$
\begin{aligned}
\boldsymbol{B}_{\ell, 0} & =\hat{\boldsymbol{r}} \frac{\ell(\ell+1)}{x^{2}} P_{\ell} H_{\ell}^{\prime \prime}+\frac{1}{x} P_{\ell}^{\prime}\left(\hat{\boldsymbol{\vartheta}} H_{\ell}^{\prime \prime \prime}-\mathrm{i} \hat{\boldsymbol{\varphi}} H_{\ell}^{\prime}\right) \\
\boldsymbol{E}_{\ell, 0} & =\frac{\omega}{q x} P_{\ell}^{\prime}\left(\hat{\boldsymbol{\vartheta}} H_{\ell}-\mathrm{i} \hat{\boldsymbol{\varphi}} H_{\ell}^{\prime \prime}\right) .
\end{aligned}
$$

We can exploit the connection between small angle cones and cylinders to estimate $\ell$. The second-order differential equation for the Legendre function becomes Bessel's equation in the small angle approximation, $\sin \vartheta \approx \vartheta$. This provides an approximation to $P_{\ell}$ which is accurate to about $1 \%$ in finding the zeros of $P_{\ell}^{\prime}$ at $\vartheta_{0}=1(\ell \approx 3)$, and improves for larger $\ell$. For:

$$
J_{0}^{\prime}=-J_{1}\left(j_{1, n}\right)=0 \quad P_{\ell}(\cos \vartheta) \cong J_{0}\left(\left[\ell+\frac{1}{2}\right] \vartheta\right) \quad \ell_{n} \cong \frac{j_{1, n}}{\vartheta_{0}}-\frac{1}{2} .
$$

Noting the appendix, equation $\left(15^{\prime} b\right)$, for $r \sim a / \vartheta_{0}$ we have $\ell / x \sim\left(j_{1, n} / \vartheta_{0}\right)\left(\vartheta_{0} / a / q\right) \sim$ $T / q$, showing directly the correspondence between the cone and cylinder, equations and eigenvalues.

\section{Solution of the ODE}

\subsection{Power-series expansion}

To develop the power-series representation of the solution to equation (17), we assume that

$$
H(x)=\sum_{n=0}^{\infty} A_{n} x^{n+s}
$$

and insert equation (20) in equation (17). For compactness, we have omitted the $\ell$ subscript of $H_{\ell}(x)$. It is assumed in equation (20) and henceforth that $H(x), A_{n}$, and $s$ depend on $\ell$, without so indicating. The requirement that $A_{0} \neq 0$ provides four possible values for $s$ :

$$
\left(s_{1}, s_{2}, s_{3}, s_{4}\right)=(\ell+3,1,0,2-\ell) .
$$

Gathering the coefficients of equal powers of $x$ and requiring that they vanish provides the recursion relation

$$
\left(n+s-s_{1}\right)\left(n+s-s_{2}\right)\left(n+s-s_{3}\right)\left(n+s-s_{4}\right) A_{n}=A_{n-4} .
$$

Setting $A_{0}=1$, we may solve for $A_{n}$ provided $\ell$ does not take on the particular values

$$
\ell=4 N+2, \quad 4 N+3, \quad \text { or } \quad 2 N-\frac{1}{2}
$$

where $N$ is a positive integer. With that restriction, we obtain the four independent solutions:

$$
H\left(s_{j}, x\right)=x^{s_{j}} \sum_{n=0}^{\infty} \prod_{\substack{i=1 \\ i \neq j}}^{4} \frac{\Gamma\left(1+\left(s_{j}-s_{i}\right) / 4\right)}{\Gamma\left(n+1+\left(s_{j}-s_{i}\right) / 4\right)} \frac{1}{n !}\left(\frac{x}{4}\right)^{4 n} .
$$


A straightforward formula for $H$ when $\ell$ takes on the values forbidden by equation (23) is available (Morse and Feshbach 1953). We will not repeat it here because, since $H$ is a continuous function of $\ell$, those values are easily avoided in any practical calculation. For the accuracy required in the graphs in figures 5 and 6 the functions are computed at $\ell=N+0.001$ instead of $\ell=N$. The series equation (24) converges in the finite $x$-plane. For large $x$, the large $n$ successive terms of each series approach every fourth term in the series for $\mathrm{e}^{x}$. Consequently, it can be shown by comparison that all four series grow exponentially.

\subsection{Asymptotic expansion}

In the $x \rightarrow \infty$ limit, equation (17) approaches the simpler fourth-order equation

$$
\frac{\mathrm{d}^{4} H}{\mathrm{~d} x^{4}}-H=0 \text {. }
$$

Four independent solutions to this equation are

$$
H(x)=\mathrm{e}^{p x}
$$

where

$$
\left(p_{1}, p_{2}, p_{3}, p_{4}\right)=(\mathrm{i},-\mathrm{i},+1,-1)
$$

are the four roots of $p^{4}=1$, corresponding to an outgoing wave, an incoming wave, exponential growth, and exponential decay, respectively.

To develop asymptotic expansions for the solutions to equation (17), first we find the solution for $p_{3}=+1$. We assume a form for the growing exponential solution, as $x \rightarrow \infty$,

$$
H(x)=\mathrm{e}^{x} w(x) \sim \mathrm{e}^{x} \sum_{n=0}^{M} B_{n} \frac{1}{x^{n}}
$$

where $(M+1)$ is an optimum number of terms used to represent $H(x)$.

Substituting equation (28) in the differential equation (17) for $H(x)$ yields the differential equation for $w(x)$,

$$
\frac{\mathrm{d}^{4} w}{\mathrm{~d} x^{4}}+4 \frac{\mathrm{d}^{3} w}{\mathrm{~d} x^{3}}+\left(6-\frac{\ell(\ell+1)}{x^{2}}\right) \frac{\mathrm{d}^{2} w}{\mathrm{~d} x^{2}}+\left(4-\frac{2 \ell(\ell+1)}{x^{2}}\right) \frac{\mathrm{d} w}{\mathrm{~d} x}-\frac{\ell(\ell+1)}{x^{2}} w=0 .
$$

Substituting the series in inverse powers of $x$ from equation (28) in equation (29), and equating terms with like powers of $x$, we get the recursion relation

$$
\begin{aligned}
& B_{0}=1 \quad B_{1}=-\frac{\ell(\ell+1)}{4} \quad B_{2}=-\frac{\ell(\ell+1)}{32}[12-\ell(\ell+1)] \\
& 4 n B_{n}=[6 n(n-1)-\ell(\ell+1)] B_{n-1}-2(n-2)[2 n(n-1)-\ell(\ell+1)] B_{n-2} \\
& -(n-2)(n-3)[n(n-1)-\ell(\ell+1)] B_{n-3} \quad \text { for } n>2 .
\end{aligned}
$$

By computing the $B_{n}$ for integer values of $\ell$ using recursion relation (30), we find that if $\ell=4 N-1$ or $4 N$ where $N=1,2,3, \ldots$, then $B_{n}=0$ for $n \geqslant \ell-1$. Consequently, for these particular values of $\ell$, the series in equation (30) terminates after a finite number of terms. For these values of $\ell$, it yields an exact solution of equation (17) in the form of a polynomial in $1 / x$ times an exponential. This solution is valid for all $x$. For other values of $\ell$, the series does not terminate, and the expansion is only asymptotic to the solution. In the computations described below, we find that truncating the asymptotic series after the $B_{n}$ term, where $n$ is the largest integer less than $\ell$, yields an accurate representation of the solution for large $x$, i.e. $x \gg \ell$. 
If $H(x)$ is a solution of equation (17), then $H(p x)$ is also a solution. Consequently, four independent asymptotic solutions of equation (17) are given by $H(p x)$. We denote these four solutions by $h_{\ell, 1}(x), h_{\ell, 2}(x), h_{\ell, 3}(x)$, and $h_{\ell, 4}(x)$, respectively. As an example, for $\ell=3$,

$$
h_{3, j}(x)=\left(1-\frac{3}{p_{j} x}\right) \mathrm{e}^{p_{j} x}
$$

and for $\ell=4$,

$$
h_{4, j}(x)=\left(1-\frac{5}{p_{j} x}+\frac{5}{p_{j}^{2} x^{2}}\right) \mathrm{e}^{p_{j} x} .
$$

Figure 2 shows the real and imaginary parts of the outgoing waves, $h_{3,1}$ and $h_{4,1}$.

Equations $(31 a)$ and $(31 b)$ indicate that all four solutions diverge at $x=0$. One can construct an outgoing wave solution that does not become infinite at the origin, using the solutions in $(31 a)$ and $(31 b)$, by taking the linear combinations

$$
\begin{aligned}
& h_{3,5}(x)=h_{3,1}-\mathrm{i} h_{3,4} \\
& h_{4,5}(x)=h_{4,1}+h_{4,4} .
\end{aligned}
$$

These are two outgoing waves for $\ell=3$ and $\ell=4$ that are finite at the origin. In fact, we will show later that these are just two special cases of the general result that

$$
h_{\ell, 5}=h_{\ell, 1}+\mathrm{e}^{\mathrm{i} \pi \ell / 2} h_{\ell, 4}
$$

is an outgoing wave, finite everywhere. In the large- $x$ asymptotic limit, $h_{\ell, 4}$ is exponentially small, and consequently $h_{\ell, 1}$ and $h_{\ell, 5}$ have the same asymptotic limit.

In figure 2 , the real and imaginary parts of $h_{\ell, 5}$ for $\ell=3$ and 4 are shown, along with the corresponding $h_{\ell, 1}$. Note that $\operatorname{Re}\left\{h_{\ell, 1}\right\}$ for $\ell=3$, and $\operatorname{Im}\left\{h_{\ell, 1}\right\}$ for $\ell=4$ are not infinite at $x=0$. This may be verified by multiplying the polynomials in equations (31a) and (31b) by the series expansions of the exponentials, and substituting in equation (31c).
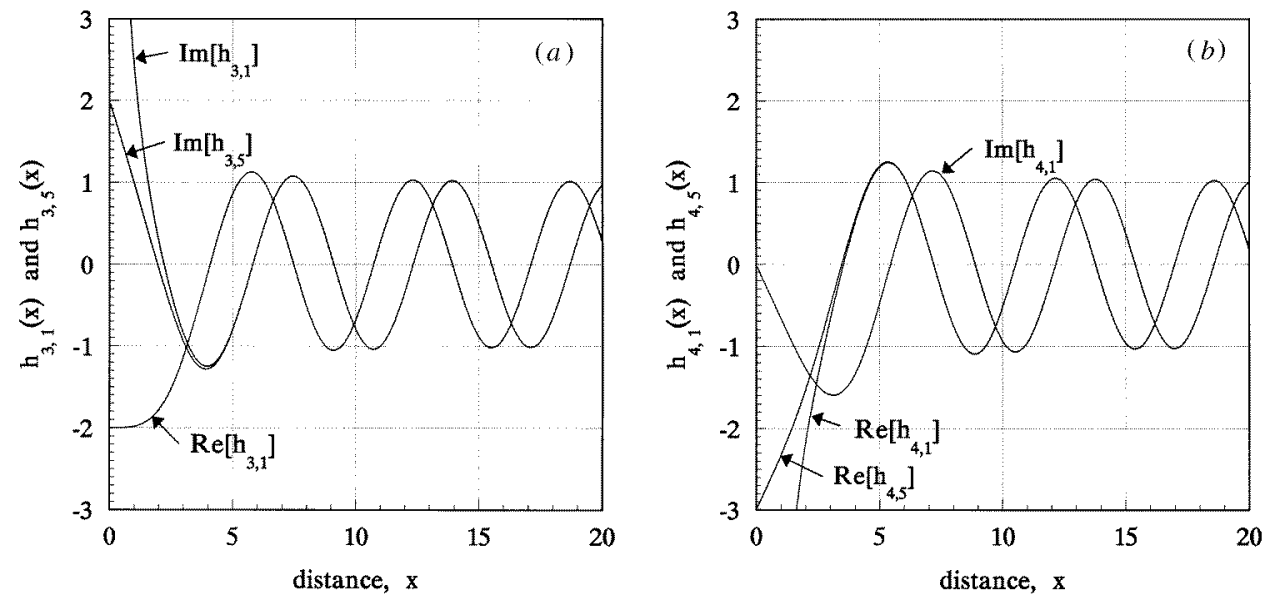

Figure 2. Exact solutions for the real and imaginary parts of the 'pure' outgoing wave, $h_{l, 1}(x)$, for $l=3$ and 4 . The exact solution for the imaginary part of the outgoing wave finite at the origin $\operatorname{Im}\left[h_{l, 5}(x)\right]$ is shown for $l=3$ and $\operatorname{Re}\left[h_{l, 5}(x)\right]$ is shown for $l=4$. For $l=3$, $\operatorname{Re}\left[h_{l, 5}(x)\right]=\operatorname{Re}\left[h_{l, 1}(x)\right]$. For $l=4, \operatorname{Im}\left[h_{l, 5}(x)\right]=\operatorname{Im}\left[h_{l, 1}(x)\right]$ 


\subsection{Integral representation of $H_{\ell}(x)$}

We start the task of obtaining an integral representation for $H_{\ell}$ by using the two series for the ordinary and modified Bessel functions of order $\ell$ (Abramowitz and Stegun 1964), and defining a new function, $\Phi(\ell, z)$, by

$$
\begin{aligned}
\Phi(\ell, z) & =\frac{1}{2}(z / 2)^{-\ell}\left[J_{\ell}(z)+I_{\ell}(z)\right] \\
& =\sum_{n=0}^{\infty} \frac{(z / 2)^{4 n}}{(2 n) ! \Gamma(2 n+\ell+1)}
\end{aligned}
$$

where the $(z / 2)^{2 n}$ terms in the $J_{\ell}$ and $I_{\ell}$ series with $n$ odd have cancelled. We will find two integral transforms of $\Phi(\ell, z)$ to transform the sum in equation (32) into the sum in equation (24).

First, we use the integral representation of the beta function and the duplication formula for the gamma function (Abramowitz and Stegun 1964) to obtain

$$
\int_{0}^{1} t^{p-1 / 2}(1-t)^{q-1 / 2} \mathrm{~d} t=\pi^{1 / 2} 2^{-2 p} \frac{\Gamma(2 p+1) \Gamma\left(q+\frac{1}{2}\right)}{\Gamma(p+1) \Gamma(p+q+1)}
$$

which is valid for $p>-\frac{1}{2}, q>-\frac{1}{2}$.

Next, we define an integral transform of $\Phi(\ell, z)$ by

$$
\begin{aligned}
\Psi(a, b, y) & =\int_{0}^{1} \Phi\left(2 a, y t^{1 / 4}\right)(1-t)^{b-1 / 2} t^{-1 / 2} \mathrm{~d} t \\
& =\sum_{n=0}^{\infty} \frac{(y / 2)^{4 n} \int_{0}^{1} t^{n-1 / 2}(1-t)^{b-1 / 2} \mathrm{~d} t}{(2 n) ! \Gamma(2 n+2 a+1)} \\
& =\pi^{1 / 2} \Gamma\left(b+\frac{1}{2}\right) \sum_{n=0}^{\infty} \frac{\left(y^{2} / 8\right)^{2 n}}{n ! \Gamma(2 n+2 a+1) \Gamma(n+b+1)}
\end{aligned}
$$

for $b>-\frac{1}{2}$. The last equality in equation (34) was obtained using equation (33), with $p=n$ and $q=b$ to evaluate the integral. In equation (34) we have eliminated the unwanted $(2 n)$ ! that was present in the denominator of equation (32), and introduced the $n$ ! and $\Gamma(n+b+1)$ needed in the denominator of equation (24).

Finally, we define an integral transform of $\Psi(a, b, y)$ by

$$
\begin{aligned}
\Omega(a, b, c, x) & =\int_{0}^{1} \Psi\left(a, b, x s^{1 / 4}\right)(1-s)^{c-a-1 / 2} s^{a-1 / 2} \mathrm{~d} s \\
& =\pi^{1 / 2} \Gamma\left(b+\frac{1}{2}\right) \sum_{n=0}^{\infty} \frac{\left(x^{2} / 8\right)^{2 n} \int_{0}^{1} s^{n+a-1 / 2}(1-s)^{c-a-1 / 2} \mathrm{~d} s}{n ! \Gamma(2 n+2 a+1) \Gamma(n+b+1)} \\
& =2^{-2 a} \pi \Gamma\left(b+\frac{1}{2}\right) \Gamma\left(c-a+\frac{1}{2}\right) \sum_{n=0}^{\infty} \frac{(x / 4)^{4 n}}{n ! \Gamma(n+a+1) \Gamma(n+b+1) \Gamma(n+c+1)}
\end{aligned}
$$

for $a>-\frac{1}{2}, b>-\frac{1}{2}, c-a>-\frac{1}{2}$. The last equality in equation (35) was obtained using equation (33), with $p=n+a$ and $q=c-a$. In equation (35) we have eliminated the $\Gamma(2 n+2 a+1)$ that was present in the denominator of equation (34), and obtained the final two gamma functions, $\Gamma(n+a+1)$ and $\Gamma(n+c+1)$, needed in the denominator of equation (24). 
For convenience, we define $\tilde{H}$, which is the sum in equation (24) without the factors that are independent of $n$ :

$$
\tilde{H}(a, b, c, x)=\sum_{n=0}^{\infty} \frac{(x / 4)^{4 n}}{n ! \Gamma(n+a+1) \Gamma(n+b+1) \Gamma(n+c+1)} .
$$

Combining equations (34) and (35), we have the double-integral representation for $\tilde{H}$,

$$
\begin{aligned}
\tilde{H}(a, b, c, x)= & \frac{(x / 4)^{-2 a}}{2 \pi \Gamma\left(b+\frac{1}{2}\right) \Gamma\left(c-a+\frac{1}{2}\right)} \int_{0}^{1} s^{a / 2-1 / 2}(1-s)^{c-a-1 / 2} \\
& \times\left(\int_{0}^{1} t^{-a / 2-1 / 2}(1-t)^{b-1 / 2}\left[J_{2 a}\left(x s^{1 / 4} t^{1 / 4}\right)+I_{2 a}\left(x s^{1 / 4} t^{1 / 4}\right)\right] \mathrm{d} t\right) \mathrm{d} s
\end{aligned}
$$

for $a>-\frac{1}{2}, b>-\frac{1}{2}, c-a>-\frac{1}{2}$.

\section{Constructing propagating and exponentiating solutions}

\subsection{Asymptotic behaviour of equation (24) for $|x| \rightarrow \infty$}

Using the integral representation of equation (37) and the known large-argument asymptotic behaviour of the Bessel functions (Abramowitz and Stegun 1964)

$$
\begin{aligned}
& J_{2 a}\left(x s^{1 / 4} t^{1 / 4}\right) \sim(\pi x / 2)^{-1 / 2} s^{-1 / 8} t^{-1 / 8} \cos \left(x s^{1 / 4} t^{1 / 4}-\left(a+\frac{1}{4}\right) \pi\right) \\
& I_{2 a}\left(x s^{1 / 4} t^{1 / 4}\right) \sim(2 \pi x)^{-1 / 2} s^{-1 / 8} t^{-1 / 8} \exp \left(x s^{1 / 4} t^{1 / 4}\right)
\end{aligned}
$$

for real arguments $x s^{1 / 4} t^{1 / 4}$ of $J_{2 a}$ and $I_{2 a}$, we can evaluate the asymptotic behaviour of equation (37) in the $x \rightarrow \infty$ limit. The contribution to the integral from the $I_{2 a}$ term grows exponentially, whereas the contribution from $J_{2 a}$ decreases with increasing $x$ because of the rapid oscillation of $J_{2 a}$. Consequently, $J_{2 a}$ will not contribute to the leading term in the asymptotic expansion and can be ignored. We have, therefore,

$$
\begin{aligned}
\tilde{H}(a, b, c, x) & \sim \frac{(2 \pi)^{-3 / 2} x^{-1 / 2}(x / 4)^{-2 a}}{\Gamma\left(b+\frac{1}{2}\right) \Gamma\left(c-a+\frac{1}{2}\right)} \int_{0}^{1} s^{a / 2-1 / 2}(1-s)^{c-a-1 / 2} \\
& \times\left(\int_{0}^{1} t^{-a / 2-1 / 2}(1-t)^{b-1 / 2} s^{-1 / 8} t^{-1 / 8} \exp \left(x s^{1 / 4} t^{1 / 4}\right) \mathrm{d} t\right) \mathrm{d} s .
\end{aligned}
$$

Furthermore, in the $x \rightarrow \infty$ limit, the major contribution to the double integral in equation (37) or (38) comes from the immediate vicinity of $s=1, t=1$. To obtain the leading term in the asymptotic expansion, we can replace $s^{a / 2-5 / 8}$ and $t^{-a / 2-5 / 8}$ by 1 . (To find higher order terms, we could expand about $s=1$ and $t=1$, but for our purposes we only need this first term.)

Making a change of variables, $s=u^{4}, t=v^{4}$, we can make the additional simplifications,

$$
\begin{aligned}
& (1-s)^{c-a-1 / 2} \rightarrow[4(1-u)]^{c-a-1 / 2} \quad \text { as } \quad u \rightarrow 1 \\
& (1-t)^{b-1 / 2} \rightarrow[4(1-v)]^{b-1 / 2} \quad \text { as } \quad v \rightarrow 1 .
\end{aligned}
$$

Introducing these changes of variables and limiting forms in equation (38), we have

$$
\begin{aligned}
\tilde{H}(a, b, c, x) \sim & \frac{\pi^{-3 / 2} 2^{2 a+2 b+2 c+1 / 2} x^{-2 a-1 / 2}}{\Gamma\left(b+\frac{1}{2}\right) \Gamma\left(c-a+\frac{1}{2}\right)} \\
& \times \int_{0}^{1}(1-u)^{c-a-1 / 2}\left(\int_{0}^{1}(1-v)^{b-1 / 2} \mathrm{e}^{x u v} \mathrm{~d} v\right) \mathrm{d} u .
\end{aligned}
$$


To obtain the asymptotic limit of equation (39), we first evaluate the asymptotic limit of the following integral in the $\beta \rightarrow \infty$ limit:

$$
\int_{0}^{1}(1-\xi)^{\alpha} \mathrm{e}^{\beta \xi} \mathrm{d} \xi=\beta^{-\alpha-1} \mathrm{e}^{\beta} \int_{0}^{\beta} w^{\alpha} \mathrm{e}^{-w} \mathrm{~d} w \rightarrow \beta^{-\alpha-1} \mathrm{e}^{\beta} \Gamma(\alpha+1)
$$

where $1-\xi=w / \beta$, and the integral representation of the gamma function is used to obtain the limit. Using equation (40) twice in succession in equation (39), as $x \rightarrow \infty$,

$$
\begin{aligned}
& \int_{0}^{1}(1-u)^{c-a-1 / 2}\left(\int_{0}^{1}(1-v)^{b-1 / 2} \mathrm{e}^{x u v} \mathrm{~d} v\right) \mathrm{d} u \\
& \rightarrow \Gamma\left(b+\frac{1}{2}\right) x^{-b-1 / 2} \int_{0}^{1}(1-u)^{c-a-1 / 2} \mathrm{e}^{x u} \mathrm{~d} u \\
& \rightarrow \Gamma\left(b+\frac{1}{2}\right) \Gamma\left(c-a+\frac{1}{2}\right) x^{a-b-c-1} \mathrm{e}^{x} .
\end{aligned}
$$

Substituting equation (41) in equation (39),

$$
\tilde{H}(a, b, c, x) \sim \pi^{-3 / 2} 2^{2 a+2 b+2 c+1 / 2} x^{-a-b-c-3 / 2} \mathrm{e}^{x} .
$$

In the derivation of equation (37), it was required that $a>-\frac{1}{2}, b>-\frac{1}{2}$, and $c-a>-\frac{1}{2}$. By making the correspondence between $a, b$ and $c$ in equation (36), and $s_{j}-s_{k}$ in equations (21) and (24), we find that the inequalities can be satisfied for $j=1,2$ and 3. But for the $s_{j}=s_{4}=2-\ell$ with $\ell>\frac{1}{2}$, no selection of $a, b$, and $c$ satisfies the inequalities, the integral representation for $H$ diverges, and the derivation of equation (39) in this case is not valid. However, by analytic continuation (Morse and Feshbach 1953) the asymptotic result of equations (41) and (42) must be valid, even in this case. This is true because equation (39) is an analytic function of $a, b$ or $c$, which we have shown agrees over a range of $a, b$ or $c$ with the leading term in the asymptotic expansion of $H_{\ell}(x)$, but is itself analytic over all $a, b, c$.

Inserting the factors in equation (24) that were omitted from equation (36), and using the result

$$
a+b+c=s-\frac{3}{2}
$$

obtained from equation (21), we have the asymptotic result as $x \rightarrow \infty$ :

$$
\begin{aligned}
& H(s, x)=C_{s} \mathrm{e}^{x} \\
& C_{s}=(2 \pi)^{-3 / 2} 2^{2 s-1} \Gamma\left(1+\frac{s-\ell-3}{4}\right) \Gamma\left(1+\frac{s-1}{4}\right) \Gamma\left(1+\frac{s}{4}\right) \Gamma\left(1+\frac{s+\ell-2}{4}\right) .
\end{aligned}
$$

\subsection{Connecting the power series and asymptotic expansion}

We now can find the four linear combinations of the four solutions to equation (24) around $x=0$ that approach $\mathrm{e}^{\mathrm{i} x}, \mathrm{e}^{-\mathrm{i} x}, \mathrm{e}^{x}$ and $\mathrm{e}^{-x}$ as $x \rightarrow+\infty$. By exploiting the symmetry properties of $H\left(s_{j}, x\right)$ in equation (24), we may generalize the result of the previous section to deduce the asymptotic behaviour as $x \rightarrow-\infty$, and $\pm \mathrm{i} \infty$ from the behaviour as $x \rightarrow+\infty$. Equation (24) may be written as

$$
H(s, x)=x^{s} F_{s}\left(x^{4}\right) \text {. }
$$

Since $F_{s}$ is a function of $x^{4}$, we see that

$$
\lim _{x \rightarrow \infty} F_{S}\left(x^{4}\right)=\lim _{x \rightarrow-\infty} F_{S}\left(x^{4}\right)=\lim _{x \rightarrow \pm i \infty} F_{S}\left(x^{4}\right)=|x|^{-s} C_{S} \mathrm{e}^{|x|}
$$


where $C_{s}$ is given by equation (43). Substituting equation (45) in equation (44),

$$
\begin{aligned}
& \lim _{x \rightarrow-\infty} H(s, x)=\lim _{x \rightarrow-\infty} x^{s}|x|^{-s} C_{s} \mathrm{e}^{|x|}=\mathrm{e}^{\mathrm{i} \pi s} C_{s} \mathrm{e}^{|x|} \\
& \lim _{x \rightarrow \pm \mathrm{i} \infty} H(s, x)=\lim _{x \rightarrow \pm \mathrm{i} \infty} x^{s}|x|^{-s} C_{s} \mathrm{e}^{|x|}=\mathrm{e}^{ \pm \mathrm{i} \pi s / 2} C_{s} \mathrm{e}^{|x|}
\end{aligned}
$$

in which we have defined the phase of $x$ in the range $-\pi<$ (phase of $x$ ) $\leqslant \pi$. Using the results in equation (46), we can write down the linear combination of the four asymptotic solutions for $|x| \rightarrow \infty$ that give the asymptotically correct $H(s, x)$ along the positive and negative real and imaginary axes:

$$
\lim _{|x| \rightarrow \infty} H(s, x)=C_{s}\left[\mathrm{e}^{-\mathrm{i} \pi s / 2} \mathrm{e}^{\mathrm{i} x}+\mathrm{e}^{\mathrm{i} \pi s / 2} \mathrm{e}^{-\mathrm{i} x}+\mathrm{e}^{x}+\mathrm{e}^{\mathrm{i} \pi s} \mathrm{e}^{-x}\right] .
$$

Denoting the analytic continuation of the four $|x| \rightarrow \infty$ solutions $\mathrm{e}^{ \pm \mathrm{i} x}$ and $\mathrm{e}^{ \pm x}$ in equation (47) by $h_{\ell, 1}(x), h_{\ell, 2}(x), h_{\ell, 3}(x)$ and $h_{\ell, 4}(x)$, respectively, and deleting the subscript $\ell$ for convenience, it follows that

$$
H(s, x)=C_{s}\left[\mathrm{e}^{-\mathrm{i} \pi s / 2} h_{1}(x)+\mathrm{e}^{\mathrm{i} \pi s / 2} h_{2}(x)+h_{3}(x)+\mathrm{e}^{\mathrm{i} \pi s} h_{4}(x)\right]
$$

where, again, $s$ can take on the four values given in equation (21). Equation (48) is a system of four equations that can be inverted to obtain $h_{1}, h_{2}, h_{3}$, and $h_{4}$, which are each a linear combination of the four functions $H\left(s_{j}, x\right), j=1,2,3,4$, and which approach $\mathrm{e}^{\mathrm{i} x}$, $\mathrm{e}^{-\mathrm{i} x}, \mathrm{e}^{x}$ and $\mathrm{e}^{-x}$, respectively, as $x \rightarrow \infty$.

Substituting the four values of $s$ in equation (48) and writing it in matrix form, we have

$$
\left[\begin{array}{c}
H(\ell+3, x) / C_{\ell+3} \\
H(1, x) / C_{1} \\
H(0, x) / C_{0} \\
H(2-\ell, x) / C_{2-\ell}
\end{array}\right]=\left[\begin{array}{cccc}
\mathrm{ie}^{-\mathrm{i} \pi \ell / 2} & -\mathrm{i} \mathrm{e}^{\mathrm{i} \pi \ell / 2} & 1 & -\mathrm{e}^{\mathrm{i} \pi \ell} \\
-\mathrm{i} & \mathrm{i} & 1 & -1 \\
1 & 1 & 1 & 1 \\
-\mathrm{e}^{\mathrm{i} \pi \ell / 2} & -\mathrm{e}^{-\mathrm{i} \pi \ell / 2} & 1 & \mathrm{e}^{-\mathrm{i} \pi \ell}
\end{array}\right]\left[\begin{array}{c}
h_{1}(x) \\
h_{2}(x) \\
h_{3}(x) \\
h_{4}(x)
\end{array}\right] .
$$

This matrix may be inverted and, after lengthy algebra, the result is

$$
h_{i}(x)=\frac{1}{D} \sum_{j=1}^{4} A_{i j} H\left(s_{j}, x\right) / C_{s_{j}}
$$

where

$$
D=8\left(\cos \pi \ell+\sin ^{3} \frac{\pi \ell}{2}+\cos ^{3} \frac{\pi \ell}{2}\right)
$$

and

$$
\left[A_{i j}\right]=\left[\begin{array}{cccc}
A & \mathrm{i} B & B & \mathrm{i} A^{*} \\
A^{*} & -\mathrm{i} B^{*} & B^{*} & -\mathrm{i} A \\
C & B & B & C^{*} \\
E & -B & B & -E
\end{array}\right]
$$

where

$$
\begin{aligned}
A & =1-\sin \pi \ell-\cos \pi \ell-2 \sin \frac{\pi \ell}{2}-\mathrm{i}\left(1-\sin \pi \ell+\cos \pi \ell+2 \cos \frac{\pi \ell}{2}\right) \\
B & =2\left(\cos \pi \ell+\sin \frac{\pi \ell}{2}+\cos \frac{\pi \ell}{2}\right) \\
E & =-2\left(1-\sin \frac{\pi \ell}{2}+\cos \frac{\pi \ell}{2}\right) \\
C & =-E(\cos \pi \ell-\mathrm{i} \sin \pi \ell) .
\end{aligned}
$$

The elements of the first row of the matrix (52), $A_{1 j}$, i.e. the coefficients for computing the outgoing wave, can be found by applying the Gaussian elimination method to 
equations (49). The other three rows can then be obtained from the first row by exploiting the symmetry properties of $H\left(s_{j}, x\right)$, equation (46), which imply equation (50), we have

$$
\lim _{x \rightarrow \infty} h_{1}(x)=\mathrm{e}^{\mathrm{i} x}=\lim _{x \rightarrow \infty}\left\{\sum_{j=1}^{4} A_{1 j} H\left(s_{j}, x\right) / C_{s_{j}}\right\} .
$$

Replacing $x$ by $-x$ in equation (35), and using equation (46), we obtain

$$
\begin{aligned}
\mathrm{e}^{-\mathrm{i} x} & =\lim _{x \rightarrow \infty}\left\{\sum_{j=1}^{4} A_{1 j} H\left(s_{j},-x\right) / C_{s_{j}}\right\} \frac{1}{D} \\
& =\lim _{x \rightarrow \infty}\left\{\sum_{j=1}^{4} A_{1 j} \mathrm{e}^{\mathrm{i} \pi s_{j}} H\left(s_{j}, x\right) / C_{s_{j}}\right\} \frac{1}{D} \\
A_{2 j} & =A_{1 j} \mathrm{e}^{\mathrm{i} \pi s_{j}} \\
A_{3 j} & =A_{1 j} \mathrm{e}^{-\mathrm{i} \pi s_{j} / 2} \\
A_{4 j} & =A_{1 j} \mathrm{e}^{\mathrm{i} \pi s_{j} / 2} .
\end{aligned}
$$

Note that if $\ell>2, h_{1}(x), h_{2}(x), h_{3}(x)$, and $h_{4}(x)$ all approach infinity as $x \rightarrow 0$, because they each contain $H\left(s_{4}, x\right)$. In particular, $h_{1}(x) \sim \mathrm{e}^{\mathrm{i} x}$ as $x \rightarrow \infty$, and $h_{1}(x) \sim$ constant $\times x^{2-\ell}$ as $x \rightarrow 0$. We can construct a linear combination of $h_{1}(x)$ and $h_{4}(x)$ that is bounded at $x=0$. And since $h_{4}(x) \sim \mathrm{e}^{-x}$ as $x \rightarrow \infty$, this combination is still asymptotically an outgoing wave as $x \rightarrow \infty$. Denoting the outgoing wave that is bounded at $x=0$ by $h_{5}(x)$, using equation (54c) with $s_{j}=2-\ell$, we have, after some manipulation,

$$
\begin{aligned}
h_{5}(x)=h_{1}(x) & -h_{4}(x) A_{14} / A_{44}=h_{1}(x)+h_{4}(x) \mathrm{e}^{\mathrm{i} \pi \ell / 2} \\
= & B\left[(1+\mathrm{i}) \frac{H_{\ell}(\ell+3, x)}{C_{\ell+3}}+\left(\cos \frac{\pi \ell}{2}-\mathrm{i}+\mathrm{i} \sin \frac{\pi \ell}{2}\right) \frac{H_{\ell}(1, x)}{C_{1}}\right. \\
& \left.-\left(1+\sin \frac{\pi \ell}{2}+\cos \frac{\pi \ell}{2}\right) \frac{H_{\ell}(0, x)}{C_{0}}\right]
\end{aligned}
$$

where $B$ is given in equation (53). Note that, of course, there is no $H_{\ell}(2-\ell, x)$ term in equation (55).

The full curve in figure 3 displays the real part of the outgoing wave $h_{\ell, 1}(x)$ for $\ell=8.5$, computed from the linear combination of the four series $H_{\ell}\left(s_{j}, x\right)$ given in equations (50)-(53). (This value of $\ell$ corresponds, see equation (19), to a cone angle of $\vartheta_{0} \cong j_{1,1} /\left(\ell+\frac{1}{2}\right)=0.43 \mathrm{rad} \equiv 24^{\circ}$.) The computation was done on a 486 microcomputer in double precision (16 digit accuracy) using a Microsoft Fortran 77 program. The gamma functions were computed using the approximation derived by Lanczos (Press et al 1994). It is seen in figure 3 that the series computation breaks down for $x \gtrsim 32$. The breakdown occurs because we are computing a linear combination of the four functions $H_{\ell}\left(s_{j}, x\right)$, each of which is proportional to $\mathrm{e}^{x}=\mathrm{e}^{32} \sim 10^{14}$, to obtain a quantity $h_{\ell, 1}(x)$ which is $\sim 1$. The broken curve is computed from the asymptotic expansion, using the recursion relation (30). It coincides with the linear combination of the $H_{\ell}\left(s_{j}, x\right)$ series over the range $2.5 \lesssim x \lesssim 32$ and extends the solution to arbitrarily large $x$. It breaks down, however, for $x \lesssim 2.5$.

Figure 4 shows the result of the same computation for the function $h_{8.5,5}(x)$, which is finite at $x=0$, obtained using equation (55), rather than for $h_{8.5,1}(x)$, which is infinite at $x=0$. For $\ell=8.5$, at small $x$ the computation using the linear combination of the $H_{\ell}\left(s_{j}, x\right)$ series is accurate, at large $x$ the computation using the asymptotic expansions is 

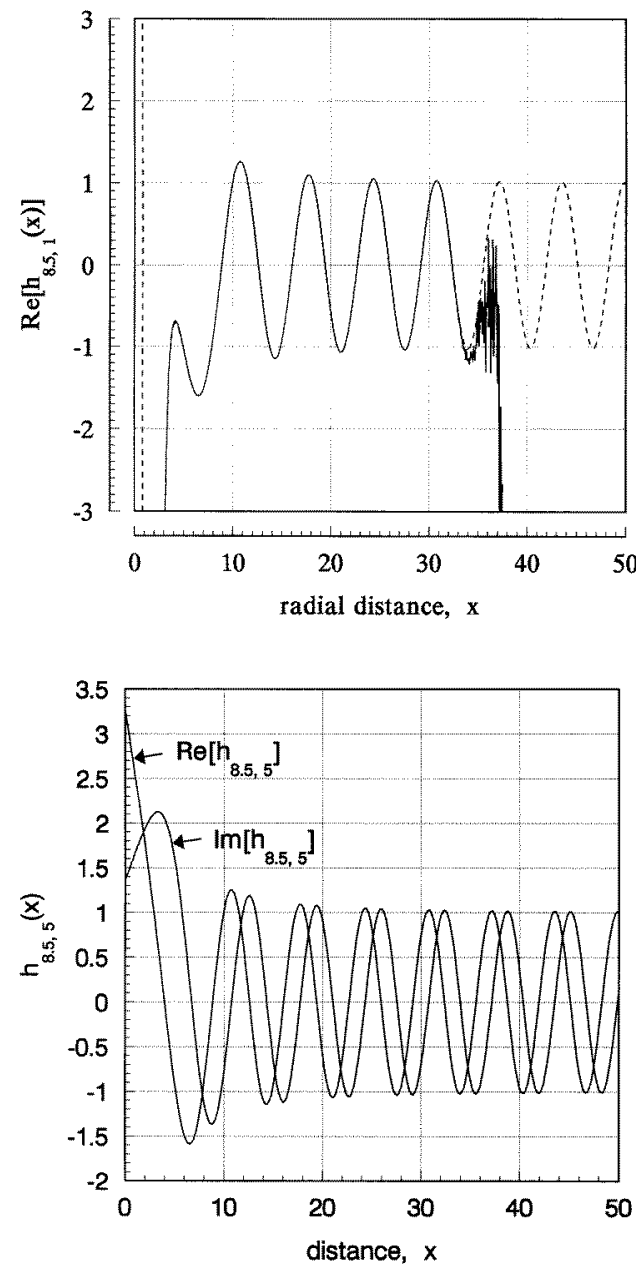

Figure 3. $\operatorname{Re}\left[h_{8,5,1}(x)\right]$ computed using the power series about $x=0$ (-) and the asymptotic series about $x \rightarrow \infty(---)$ illustrating the breakdown of the numerical procedure using the power series for $x \gtrsim 32$.

Figure 4. $\operatorname{Re}\left[h_{8,5,1}(x)\right]$ computed using the power series for $0 \leqslant x<20$ and the asymptotic series for $20 \leqslant x \leqslant 50$.

accurate, and for a very large intermediate range $2.5 \lesssim x \lesssim 32$, they are both accurate representations of the solution. We have illustrated the computations in figures 2 and 3 for $\ell=8.5$ in order to compare the computation using equations (31)-(34) and (38) for $\ell=8.5$ with the computation using equations (47) and (49), shown previously in figure $1(d)$, for the comparable value $\ell=8$. The results shown in figures 2 and 3 have the same general form as the results for $h_{8,1}(x)$ and $h_{8,5}(x)$ in figure $1(d)$, as one would expect. Below, we will show the dependence on $\ell$ over a wide range.

It should be noted that, although we have an exact solution in the form of a finite polynomial times an exponential for special values of $\ell=3,4,7,8,11,12, \ldots$, the computation of $h_{\ell, 5}(x)$ from these solutions at small $x$ and large $\ell$ presents computational difficulties. The finite value of these functions $h_{\ell, 5}(x)$ at $x=0$ is the result of a delicate cancellation of large terms in the polynomial-exponential product solutions $h_{\ell, 1}$ and $h_{\ell, 4}$. Consequently, the linear combination of the four infinite series $H_{\ell}\left(s_{j}, x\right)$ is the method of choice for computing $h_{\ell, 5}(x)$ for large $\ell$ and small $x$.

For the most general problem that can be approximated by a sequence of contiguous segments of truncated cones, as described in the introduction, all four solutions $H_{\ell}\left(s_{j}, x\right)$, $j=1,2,3,4$, are needed to satisfy boundary conditions between adjacent conical segments 
(or, equivalently, all four solutions $h_{\ell, j}(x)$ ). We have not shown the results of numerical computations of the functions $H_{\ell}\left(s_{j}, x\right)$ because they are not very informative. The oscillations that we have seen in the solutions as a function of $x$, although obviously present since they emerge after performing the appropriate linear combinations, are totally obscured in the individual $H\left(s_{j}, x\right)$ functions by the exponential growth present in each function.

In figure 5, the real and imaginary parts of the outgoing wave that is finite at $x=0$, $h_{\ell, 5}(x)$ are shown for $0 \leqslant \ell \leqslant 28$ and $0 \leqslant x \leqslant 30$. The computations were done using the linear combination (55) of the four series $H_{\ell}\left(s_{j}, x\right)$ for $0<x \leqslant 20$ and the asymptotic expansion for $20<x \leqslant 28$. We see that for $\ell=0$, the function settles down to its asymptotic form $\mathrm{e}^{\mathrm{i} x}$ very quickly. (The solution for $\ell=0$ is just $h_{0,5}(x)=\mathrm{e}^{\mathrm{i} x}+\mathrm{e}^{-x}$.) For increasing $\ell$, it also approaches $\mathrm{e}^{\mathrm{i} x}$, but only after oscillation-free intervals of $x$ which increase in length as $\ell$ increases (much like the behaviour of the Bessel function $J_{\ell}(x)$, for example, except that in the present case the oscillation is undamped). There is a $\pi / 2$ phase difference between the oscillation of the real and imaginary parts. In figure 6 , the real and imaginary parts of $g_{\ell, 5}(x) \equiv \mathrm{d}^{2} h_{\ell, 5}(x) / \mathrm{d} x^{2}$ are shown. According to equation (18), $g_{\ell, 5}(x)$ is required for calculating the radial component (along the axis of the cone) of the magnetic field, and together with $h_{\ell, 5}(x)$ for calculating the two components (transverse to the axis) of the electric field.

\subsection{WKB solution to equation (17)}

In the usual manner (see, for example, Swanson 1989) a WKB solution to equation (17) can be obtained by assuming the form

$$
H(x)=A(x) \exp (\mathrm{i} \phi(x))=A(x) \exp \left(\mathrm{i} \int_{x_{0}}^{x} k(y) \mathrm{d} y\right)
$$

where $x_{0}$ is an arbitrary location where the phase is chosen to be zero and $k$ is its wavenumber divided by $q . A(x)$ and $k(x)$ are assumed to be slowly varying functions, so that we can neglect (using a prime to denote derivative with respect to $x$ ) $A^{\prime \prime}, k^{\prime \prime}, k^{\prime} A^{\prime}$, and $\left(k^{\prime}\right)^{2}$, with respect to $A, k, A^{\prime}$ and $k^{\prime}$. Inserting equation (56) in equation (17) and requiring that both the real and imaginary parts of the equation be satisfied, we get

$$
\begin{aligned}
& k^{4}+U^{2} k^{2}-1=0 \\
& 2 k\left(2 k^{2}+U^{2}\right) A^{\prime}+\left(6 k^{2}+U^{2}\right) k^{\prime} A=0
\end{aligned}
$$

where $U^{2}(x)=\ell(\ell+1) / x^{2}$ corresponds to $T^{2}$ in the cylindrical case. We choose the propagating roots of equation (57):

$$
k(x)= \pm\left[\frac{1}{2}\left(\sqrt{U^{4}(x)+4}-U^{2}(x)\right)\right]^{1 / 2} .
$$

Differentiating equation (57) and inserting the result in equation (58), we get

$$
\begin{aligned}
& \frac{k^{\prime}}{k}=-\frac{1}{2\left(2 k^{2}+U^{2}\right)^{2}} \frac{\mathrm{d} U^{2}}{\mathrm{~d} x} \\
& \frac{A^{\prime}}{A}=\frac{1}{4} \frac{6 k^{2}+U^{2}}{\left(2 k^{2}+U^{2}\right)^{2}} \frac{\mathrm{d} U^{2}}{\mathrm{~d} x}=\frac{1}{4} \frac{3 \sqrt{U^{4}+4}-2 U^{2}}{U^{4}+4} \frac{\mathrm{d} U^{2}}{\mathrm{~d} x}
\end{aligned}
$$

which can be integrated exactly to yield

$$
A(x)=\frac{\left[U^{2}(x)+\sqrt{U^{4}(x)+4}\right]^{3 / 4}}{\left[2\left(U^{4}(x)+4\right)\right]^{1 / 4}} .
$$



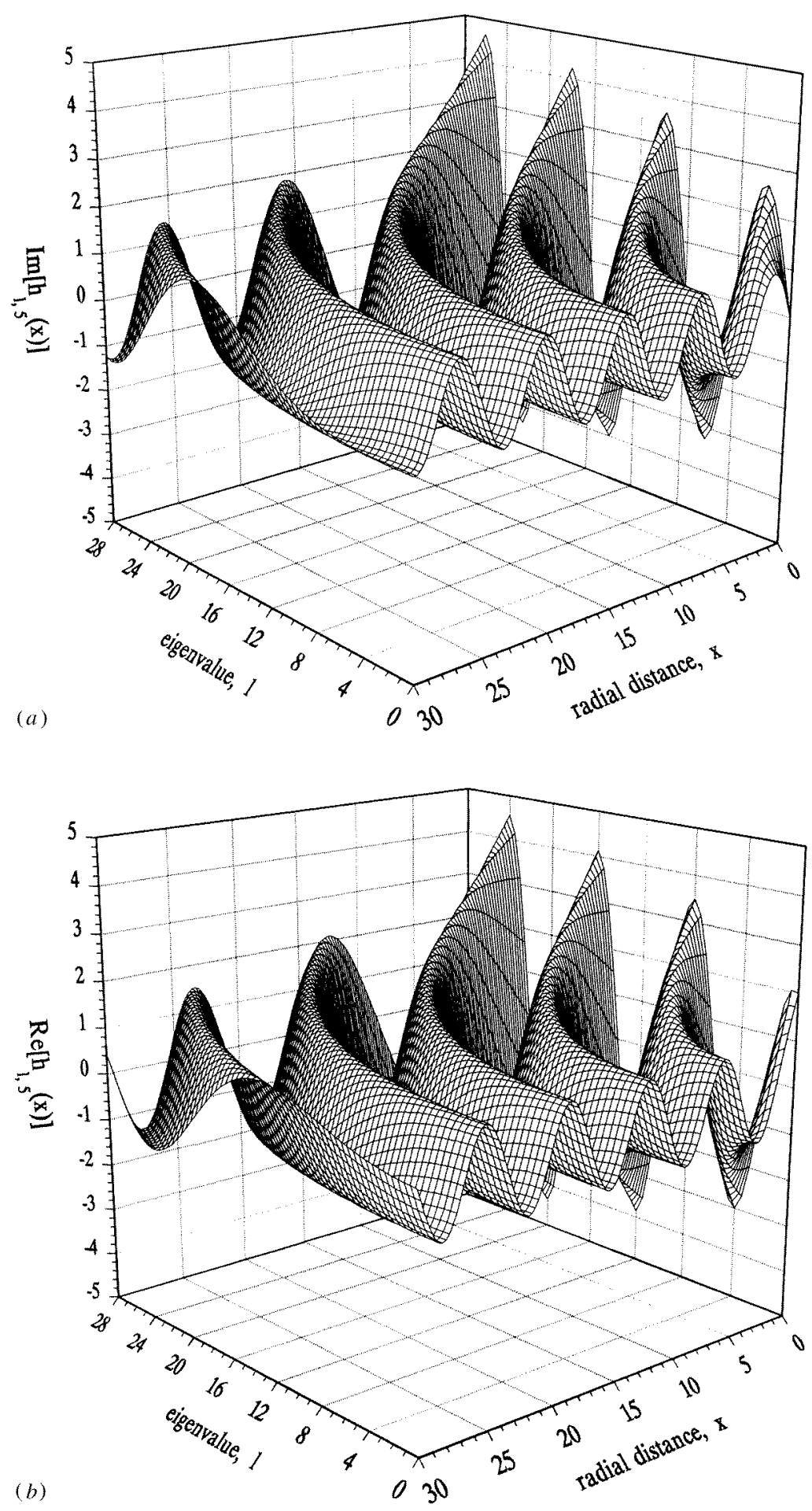

Figure 5. The real $(a)$ and imaginary $(b)$ parts of $h_{l, 5}(x)$, as a function of $l$ and $x$ computed using the power series for $0<x \leqslant 20$ and the asymptotic series for $20 \leqslant x \leqslant 28$. 

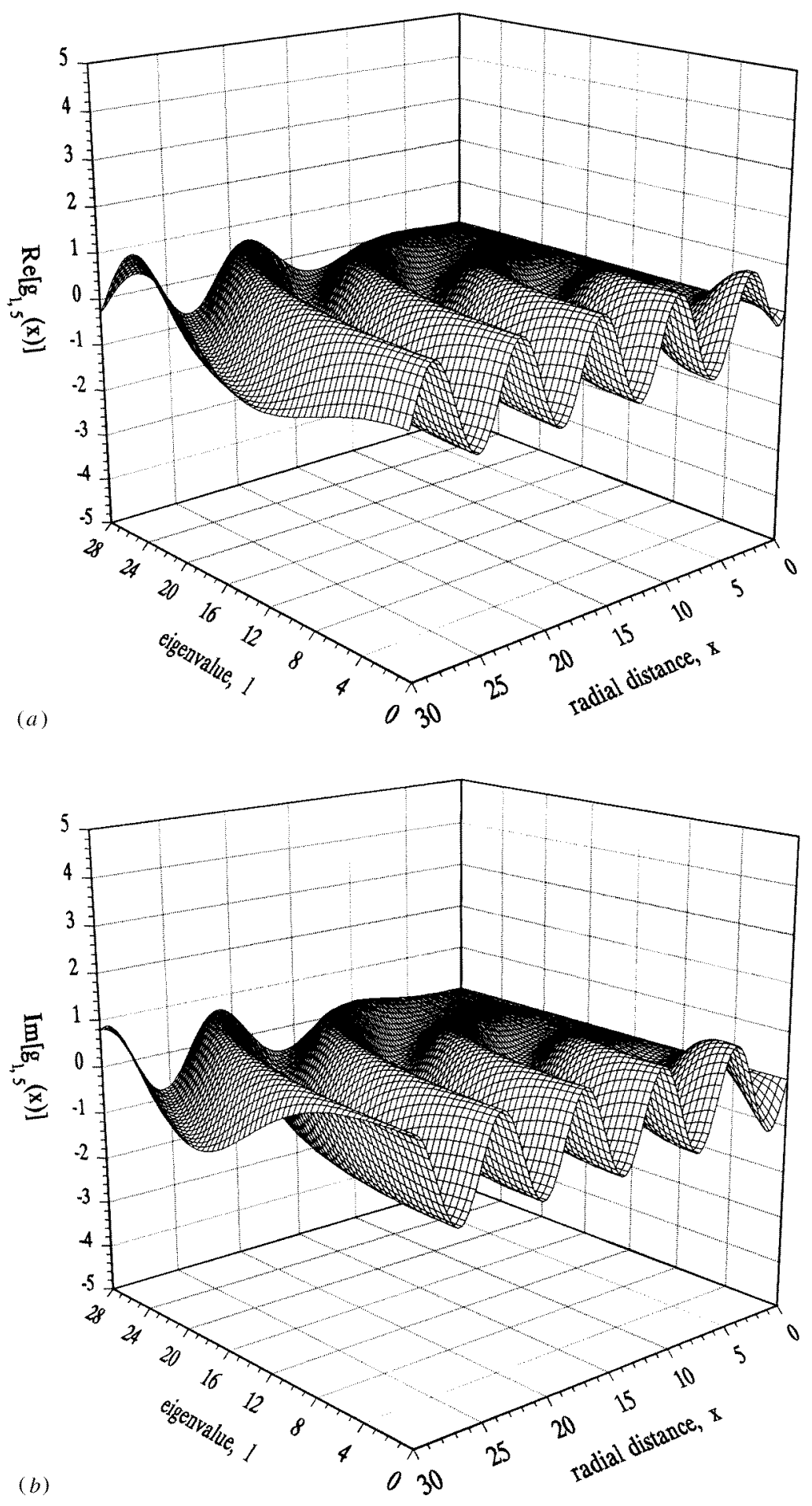

Figure 6. The real (a) and imaginary $(b)$ parts of $g_{l, 5}(x)$ computed as in figure 5. 
Thus, the form of equation (56) with the phase obtained from equation (59) is analogous to the WKB solution for the case of a 1D inhomogeneous dielectric where the equation for the field is second order. However, the amplitude in equation (61) differs significantly from the second-order result, $A=1 /(k)^{1 / 2}$, because of the $2 \mathrm{D}$ boundary constraints and the helicon coupling which requires that the fields satisfy a fourth-order equation.

It is convenient, particularly in view of the exact solutions obtained above, to fix the phase of the WKB approximation so that

$$
\phi(x)=\int_{x_{0}}^{x} k(y) \mathrm{d} y=x+I(x)
$$

where $I(x)$ vanishes at infinity. Taking $x_{0}$ arbitrarily large, subtracting the arbitrarily large constant, and changing variables, we obtain

$$
\begin{array}{r}
\phi(x)=x+\frac{\sqrt{\ell(\ell+1)}}{2} \int_{0}^{U(x)} \frac{\mathrm{d} y}{y^{3 / 2}}\left\{1-\left[\frac{\sqrt{x^{2}+4}-x}{2}\right]^{1 / 2}\right\} \\
\underset{x \rightarrow \infty}{\rightarrow} x\left[1+\frac{1}{4} U(x)-\frac{1}{96} U^{2}(x)-\frac{1}{160} U^{3}(x)+\cdots\right] .
\end{array}
$$

Thus, the WKB approximation provides an excellent bridge to the cylindrical limit $x \rightarrow \infty$, $U(x) \rightarrow T$ (see the appendix).

The WKB approximation to equation (17) is valid for

$$
W_{w}(\ell, x)=\left.\frac{1}{k^{2}} \frac{\partial k}{\partial x}\right|_{\ell=\mathrm{constant}}=\frac{U^{2}}{x k \sqrt{U^{4}+4}} \ll 1 .
$$

The ratio of the WKB to the exact amplitude is shown as a function of $x$ and $\ell$ in figure 7(a), and the difference between the WKB and exact phases in figure 7(b). If, for example, a wave propagates from $x_{1}$ to $x_{2}$, the ratio of the magnitude of the $\mathrm{WKB}$ to exact solutions is given by the ratio of the projection in figure $7(a)$ at $x_{2}$ to that at $x_{1}$. Similarly, the difference between the WKB and exact phases is given by the projection in figure $7(b)$ at $x_{2}$ minus that at $x_{1}$. The contour plot projections shown at the bottom of the figures illustrate the fact that the WKB approximation is excellent for many cases of physical interest.

The fields in this approximation are given by equations (18) with

$$
\begin{aligned}
& \frac{H^{\prime}}{H}=\mathrm{i} k-\frac{6 k^{2}+U^{2}}{U^{4}+4}\left(\frac{U^{2}}{2 x}\right) \\
& \frac{H^{\prime \prime}}{H}=-k^{2}\left[1+\mathrm{i} \frac{4 k}{x} \frac{U^{2}}{U^{4}+4}\right] \\
& \frac{H^{\prime \prime \prime}}{H}=-\mathrm{i} k^{3}+\frac{3}{2} \frac{k^{2} U^{2}}{x} \frac{2 k^{2}-U^{2}}{U^{4}+4} .
\end{aligned}
$$



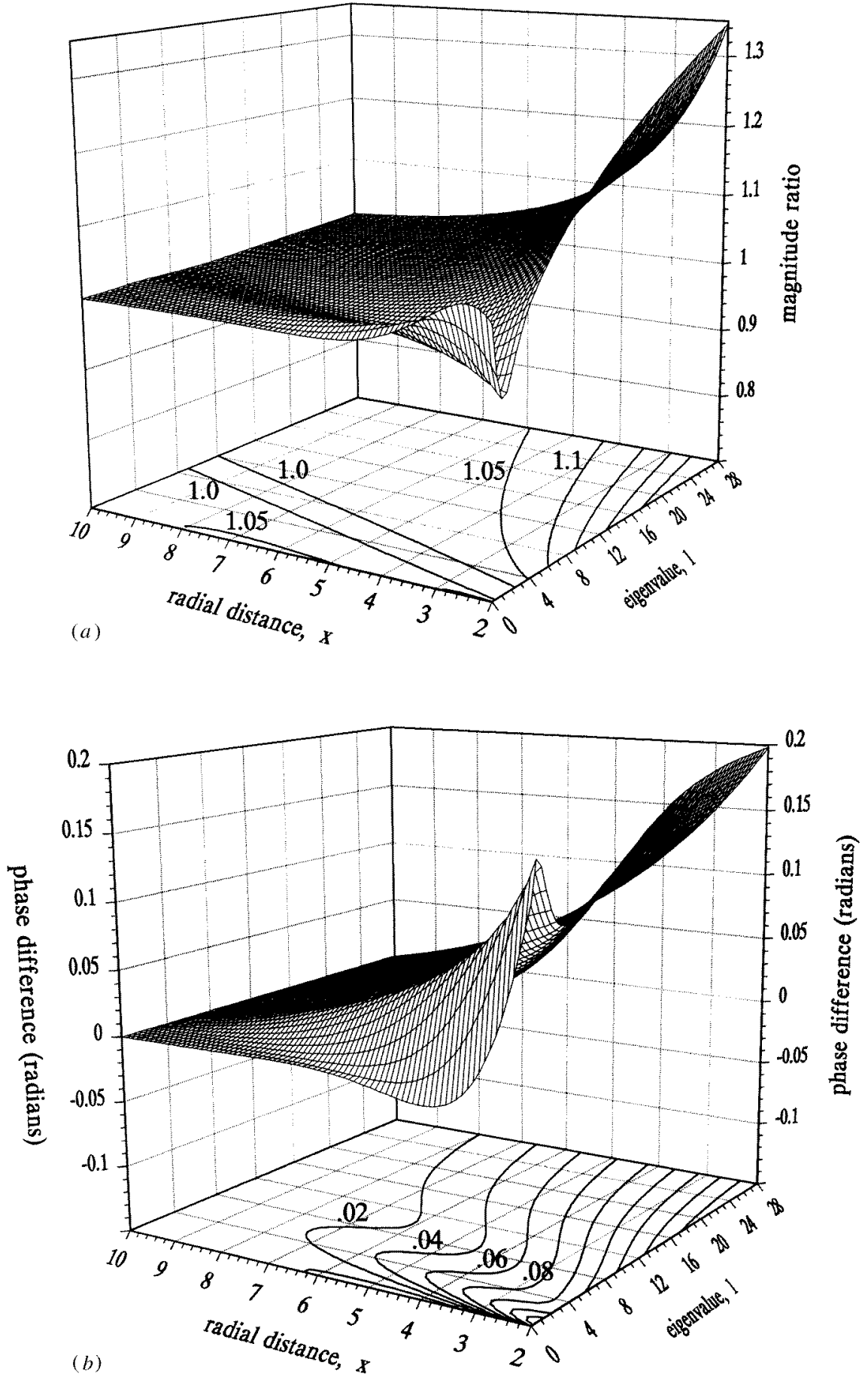

Figure 7. (a) The ratio of the magnitude of the WKB to exact solutions. Contours of constant ratio are shown at the bottom. (b) The difference between the WKB and exact solution phases. Constant phase difference contours are shown at the bottom. 


\section{Propagation in a slowly flaring guide field}

In this section, as an illustration of the properties of the conical helicon functions, we investigate the case of propagation of the fundamental $\ell$-mode in a parabolically flaring field. We assume that the flare is sufficiently gradual for the influence of reflected and exponential waves and higher $\ell$-mode generation to be negligible, and consider only cases for which $x$ is large enough for the WKB approximation to be valid.

Consider the geometry of figure 1 . Let $h(\ell, x)$ be the outgoing wave solution in a cone, normalized to unit amplitude at $x=\infty$, and the solution throughout the entire actual bounded region be $H(z)$, where $z$ is the distance measured along the axis of the region from the junction of the cylinder and the parabola. In the $n$th finite element of the computation, the $\ell$ value will be fixed by the angle made by the line $\left(A_{n}, b_{n}\right)$ and the $z$-axis, and $x$ will vary. As before, $x$ is the non-dimensional radial distance measured from the apex of the cone, $A_{n}$. Note that the $x$ variable is individualized for each finite element. Let the $\ell$ value be identified by the $z$ value at the left-hand surface. Thus, in region 1 , where $z_{0}<z<z_{1}$, we have for $H\left(z_{0}\right)=1$, i.e. unit amplitude at the initial point

$$
H(z)=\frac{h\left[\ell\left(z_{0}\right), x(z)\right]}{h\left[\ell\left(z_{0}\right), x\left(z_{0}\right)\right]} .
$$

Define the quantities

$$
H_{n}=H\left(z_{n}\right) \quad \text { and } \quad h_{m, n}=h\left[\ell\left(z_{m}\right), x\left(z_{n}\right)\right] .
$$

Hence, $H_{1}=h_{0,1} / h_{0,0}$. In region 2 , where $z_{1}<z<z_{2}$,

$$
H(z)=H_{1} \frac{h\left[\ell\left(z_{1}\right), x(z)\right]}{h\left[\ell\left(z_{1}\right), x\left(z_{1}\right)\right]}
$$

and

$$
H_{2}=H_{1} \frac{h_{1,2}}{h_{1,1}}=\frac{h_{0,1}}{h_{0,0}} \frac{h_{1,2}}{h_{1,1}} .
$$

By induction, we have

$$
H\left(z_{n+1}\right)=H_{n+1}=H_{n} \frac{h_{n, n+1}}{h_{n, n}}=\prod_{j=0}^{n} \frac{h\left[\ell\left(z_{j}\right), x\left(z_{j+1}\right)\right]}{h\left[\ell\left(z_{j}\right), x\left(z_{j}\right)\right]} .
$$

Equation (69) is the basis of the numerical calculation to follow. It is of interest to write this result analytically, by passing to the limit of infinitesimal truncated cones. From equation (69), we have

$$
\frac{H_{n+1}-H_{n}}{H_{n}}=\frac{h_{n, n+1}-h_{n, n}}{h_{n, n}}
$$

which we rewrite as

$$
\frac{1}{H} \frac{\Delta H}{\Delta z}=\left.\frac{1}{h} \frac{\Delta_{x} h}{\Delta x} \frac{\Delta x}{\Delta z}\right|_{\ell=\text { constant }} .
$$

If the tangent angle of the bounding curve is $\vartheta$, then in the infinitesimal limit $\mathrm{d} x / \mathrm{d} z=\cos \vartheta$, and we have

$$
\ln [H(z)]=\left.\int_{0}^{z} \mathrm{~d} z^{\prime}\left[\cos \vartheta\left(z^{\prime}\right) \frac{\partial}{\partial x} \ln \{h(\ell, x)\}\right]\right|_{\ell=\ell\left(z^{\prime}\right), x=x\left(z^{\prime}\right)} .
$$



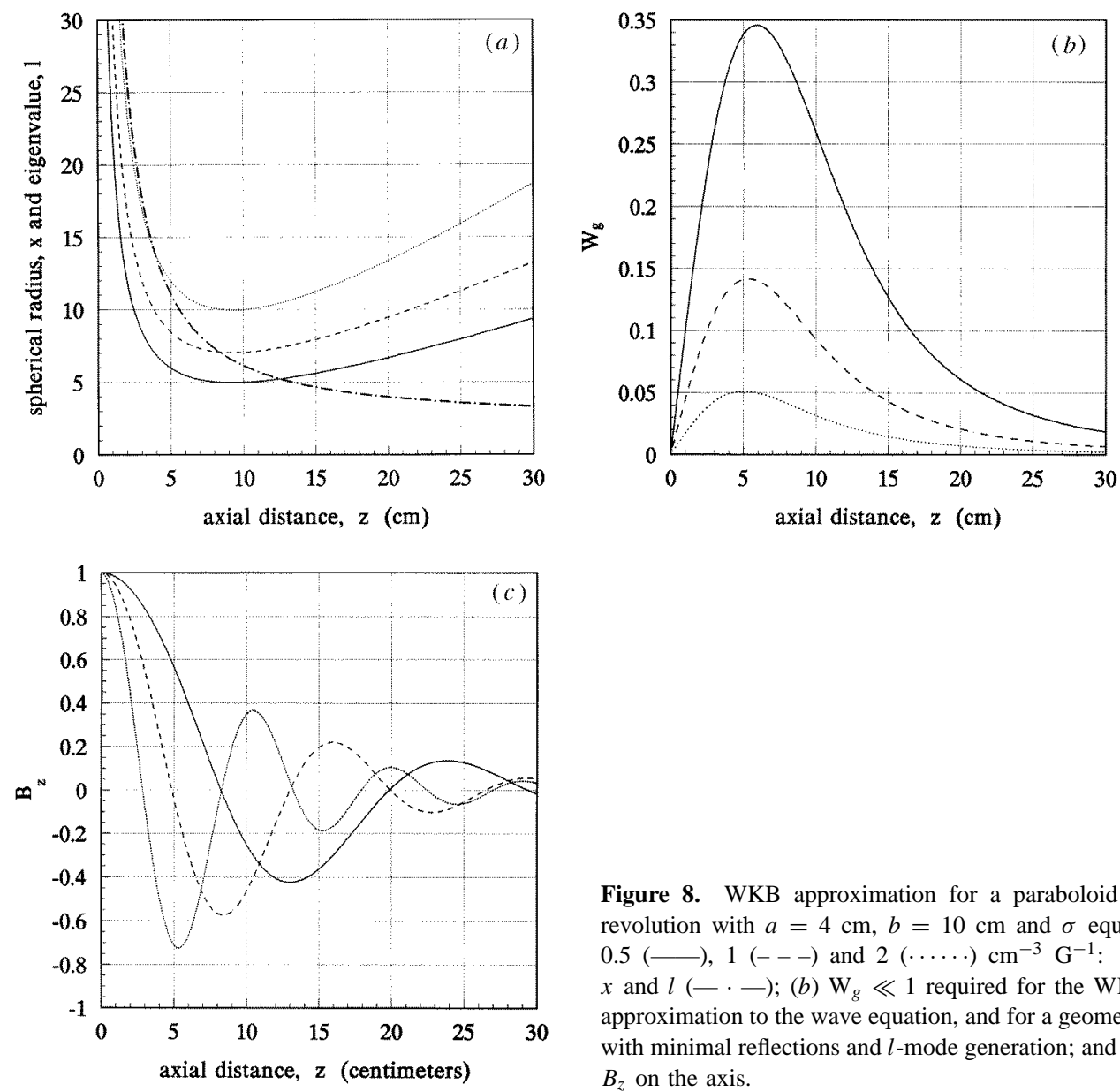

Figure 8. WKB approximation for a paraboloid of revolution with $a=4 \mathrm{~cm}, b=10 \mathrm{~cm}$ and $\sigma$ equals 0.5 (-), $1(---)$ and $2(\cdots \cdots) \mathrm{cm}^{-3} \mathrm{G}^{-1}:(a)$ $x$ and $l(-.-) ;(b) \mathrm{W}_{g} \ll 1$ required for the WKB approximation to the wave equation, and for a geometry with minimal reflections and $l$-mode generation; and $(c)$ $B_{z}$ on the axis.

Propagation was calculated for helicon waves incident from the left at $z=0$ into a region where the bounding magnetic field lines form a parabola of revolution with a cylindrical radius

$$
\rho\left(z_{b}\right)=a\left(1+z_{b}^{2} / b^{2}\right)
$$

where $z_{b}$ is the projection of a boundary point on the axis. We use the condition

$$
W_{g}=\frac{1}{k^{2} q} \frac{\mathrm{d} k}{\mathrm{~d} z_{b}} \ll 1
$$

to determine if the flare of the guide field is sufficiently gentle to neglect reflections and higher order $\ell$-mode coupling, and use the WKB approximation, equation (56), for the propagating cone function $h(\ell, x)$. The plasma parameters were characterized using

$$
\sigma=10^{-10} \frac{n}{B} \mathrm{~cm}^{-3} \mathrm{G}^{-1}
$$

and $\omega / 2 \pi=27 \mathrm{MHz}$. Values of $\sigma$ of $0.5,1$ and $2 \mathrm{~cm}^{-3} \mathrm{G}^{-1}$ were used. They correspond, for example, to $B_{0}=1 \mathrm{kG}$ and $n_{0}$ equals $5 \times 10^{12}, 10^{13}$ and $2 \times 10^{13} \mathrm{~cm}^{-3}$. The six combinations consisting of $a$ equals 1,2 and $4 \mathrm{~cm}$ and $b$ equals 10 and $20 \mathrm{~cm}$ were investigated. The configuration for calculating $\ell$ and $x$ as a function of $z$ is shown in figure 1 for $a=4 \mathrm{~cm}$ and 
$b=10 \mathrm{~cm}$. The values of $z$ were limited to $0 \leqslant z \leqslant z_{\max }$, where $z_{\max }$ is selected to insure that $f \ll f_{\text {ce. }}$. Values of $\ell$ and $x$ for $a=4 \mathrm{~cm}$ and $b=10 \mathrm{~cm}$ are presented in figure 8(a) for $\sigma$ equals $0.5,1$ and $2 \mathrm{~cm}^{-3} \mathrm{G}^{-1}$. It shows a monotonic decrease of and a broad minimum of $x$ at about $8 \mathrm{~cm}$. For these cases, using equation (59), the wavelengths in the cylinder are $35.8,18.7$ and $10.4 \mathrm{~cm}$, and the wavelengths in a fully expanded guide (i.e. the whistler wavelengths, $2 \pi / q$ ) are $15.2,10.8$ and $7.6 \mathrm{~cm}$, respectively. The measure of reflection and $\ell$-mode generation, $W_{g}$, which was found to be slightly larger than $W_{w}$, is shown in figure $8(b)$ for that same case. It shows a peak at about $5 \mathrm{~cm}$ for each of the three densities, with the largest peak at the lowest density (for which the wavelength must undergo the largest contraction from the cylinder to infinity). With $W_{g}$ and $W_{w} \ll 1$ over most of the range for all three densities we expect the WKB approximation to well represent the fields. The actual errors incurred can be estimated from figure 7. The shape of the $W_{g}$ curve is similar for all six combinations of $a$ and $b$. For the radius doubling distance, $b=20 \mathrm{~cm}$, and $a=2 \mathrm{~cm}$, and particularly for $a=4 \mathrm{~cm}$, the WKB criterion is similarly met for all three values of the density. For $a=1 \mathrm{~cm}$ and $b=10 \mathrm{~cm} W_{g} \ll 1$ for a substantial portion of the range only for the highest density $\left(\sigma=2 \mathrm{~cm}^{-3} \mathrm{G}^{-1}\right)$. At lower densities it is necessary to include both reflections and $\ell$-mode generation, use the four exact solutions of the wave equation and solve the resulting large number of simultaneous equations. Similarly, for $a=1 \mathrm{~cm}$ and $b=20 \mathrm{~cm}$, and for $a=2 \mathrm{~cm}$ and $b=10 \mathrm{~cm}$, the WKB criterion is substantially met for $\sigma$ equals 1 and $2 \mathrm{~cm}^{-3} \mathrm{G}^{-1}$, but not at the lowest density. Thus, the WKB condition is satisfied for the 'gentler geometries' in combination with the smaller changes in the wavelength. The value of $B_{z}$, calculated on the axis, is shown for $a=4 \mathrm{~cm}$ and $b=10 \mathrm{~cm}$ in figure $8(c)$. Reduction of the amplitude as the wave propagates into a guide of increasing radius, necessary for energy conservation, is apparent at all three densities. Decrease of the apparent wavelength is clearly visible at the lowest density and barely discernible at the highest.

\section{Acknowledgment}

The authors thank Professor F F Chen for suggesting this problem and for many helpful discussions.

\section{Appendix. Helicon waves in a cylinder}

To analyse equation (10) further, it is instructive to consider propagation in a cylinder using the multipolar potential formulation. In that case, use equation (7) with the substitution $\hat{\boldsymbol{r}} r \rightarrow \hat{z}$. The separation proceeds as in equations (8) and (9), resulting in equations (10'), with the derivatives with respect to $z$ rather than $r$, and the factor $r$ everywhere replaced by 1 :

$$
\begin{aligned}
& k_{0}^{2} \psi=\frac{\partial}{\partial z}\left(\mathrm{i} \beta_{1} \nabla^{2} \chi\right)+\beta_{2} \nabla^{2} \psi+\frac{\mathrm{d} \beta_{2}}{\mathrm{~d} z} \frac{\partial \psi}{\partial z}-\beta_{3}\left[\nabla^{2} \psi-\frac{\partial^{2} \psi}{\partial z^{2}}\right] \\
& k_{0}^{2} \chi=-\mathrm{i} \beta_{1} \frac{\partial \psi}{\partial z}+\beta_{2} \nabla^{2} \chi .
\end{aligned}
$$

To expand in eigenfunctions, use in place of equation (11)

$$
\psi=\sum_{T, m} F_{T, m}(z) J_{m}(T \rho) \exp (\mathrm{i} m \varphi)
$$

and

$$
\chi=\sum_{T, m} G_{T, m}(z) J_{m}(T \rho) \exp (\mathrm{i} m \varphi)
$$


where the $T$ 's are the radial separation constants, and $J_{m}$ is the Bessel function. The azimuthal eigenvalue $m$ is an integer, as in the conical case. For the zero radial current at the boundary of the cylinder $\rho=a$, we have in place of equations (12), (13), and (14), suppressing the subscript $m$ (except in $J_{m}$ ):

$D_{T}^{2}=\frac{\mathrm{d}^{2}}{\mathrm{~d} z^{2}}-T^{2}$

$\sum_{T}\left\{k_{0}^{2} F_{T}-\beta_{2} D_{T}^{2} F_{T}-\beta_{2}^{\prime} F_{T}^{\prime}-\beta_{3} T^{2} F_{T}-\left(\mathrm{i} \beta_{1} D_{T}^{2} G_{T}\right)^{\prime}\right\} J_{m}=0$

$\sum_{T}\left\{k_{0}^{2} G_{T}-\beta_{2} D_{T}^{2} G_{T}+\mathrm{i} \beta_{1} F_{T}^{\prime}\right\} J_{m}=0$

$\mu_{0} J_{r}=\left.\sum_{T}\left\{\left[\left(1+\beta_{2}\right) J_{m}^{\prime}-\frac{m}{\rho} \beta_{1} J_{m}\right] F_{T}^{\prime}+\mathrm{i}\left[\beta_{1} J_{m}^{\prime}-\left(1+\beta_{2}\right) \frac{m}{\rho} J_{m}\right] D_{T}^{2} G_{T}\right\}\right|_{\rho=a}=0$

where $J_{m}^{\prime}$ is the derivative of $J_{m}$ with respect to $\rho$, and the primes of $F$ and $G$ denote derivatives with respect to $z$. If the $\beta$ 's are independent of $z, F$ and $G$ are proportional to $\exp (\mathrm{i} k z)$ and satisfy equations $\left(13^{\prime}\right)$ and $\left(14^{\prime}\right)$ for each $T$ in the summation for all $z$. Otherwise, as in the case of the cone, equation $\left(14^{\prime}\right)$ can only be satisfied for each $T$ if $m=0$. In the latter case, equation $(15 b)$ becomes

$$
J_{0}^{\prime}(T a)=0
$$

which yields a dispersion relation which is biquadratic in both $T^{2}$ and $k^{2}$ :

$$
k^{2}\left(k^{2}+T^{2}\right) \beta_{1}^{2}-\left[k_{0}^{2}+\beta_{2}\left(k^{2}+T^{2}\right)\right]\left[k_{0}^{2}+\beta_{2}\left(k^{2}+T^{2}\right)-\beta_{3} T^{2}\right]=0
$$

which is a generalization of the familiar result of Chen (1991), $k^{2}+T^{2}=\alpha^{2} / k^{2}=$ $\left(k_{0}^{2} / \beta_{1}\right)^{2} / k^{2}$, for $\beta_{2}=\beta_{3}=0$. Combining equations $\left(13^{\prime}\right)$ and $\left(14^{\prime}\right)$ provides unique solutions for $T^{2}$ and $k^{2}$, which may in general be complex. In the case $m=0$, where equation $\left(15^{\prime} b\right)$ obtains, $T$ is real, and for the parameter regime of equation $(1), k^{2}$ has a positive and a negative root corresponding to propagating and exponentiating solutions. In propagation through a flaring magnetic field, calculated for example by the methods of section 4 , the previously unidentified exponentiating solutions can play a small but crucial role in the self-consistent treatment of helicon waves.

\section{References}

Abramowitz M and Stegun I A 1964 Handbook of Mathematical Functions National Bureau of Standards (Washington, DC: US Govt Printing Office)

1994 Helicon plasma sources High Density Plasma Sources ed L A Popov (Noyes)

Chen F F 1991 Plasma Phys. Control. Fusion 33 3399-401

Dahlquist G and Björck A (translated by Anderson N) 1974 Numerical Methods (Englewood Cliffs, NJ: PrenticeHall)

Morse P M and Feshbach H 1953 Methods of Theoretical Physics (New York: McGraw-Hill)

Press W H, Flannery B P, Teukolsky S A and Vetterling W T 1994 Numerical Recipes, the Art of Scientific Computing (Cambridge: Cambridge University Press)

Solymar L 1959 IEE Proc. B 106 121-8

Sporleder F and Unger H-G 1979 Waveguide tapers, transitions, and couplers IEEE Electromagnetic Waves Series 6 ed J R Wait et al (London: IEEE)

Stix T H 1992 Waves in Plasmas (New York: American Institute of Physics)

Swanson D G 1989 Plasma Waves (London: Academic) 